VOL. 63 (2001) [407-433]

\title{
THE PSEUDOIDENTITY PROBLEM AND REDUCIBILITY FOR COMPLETELY REGULAR SEMIGROUPS
}

\author{
Jorge Almeida AND Peter G. Trotter
}

\section{Dedicated to George Szekeres on the occasion of his 90th birthday}

\begin{abstract}
Necessary and sufficient conditions for equality over the pseudovariety CR of all finite completely regular semigroups are obtained. They are inspired by the solution of the word problem for free completely regular semigroups and clarify the role played by groups in the structure of such semigroups. A strengthened version of Ash's inevitability theorem ( $\kappa$-reducibility of the pseudovariety $\mathbf{G}$ of all finite groups) is proposed as an open problem and it is shown that, if this stronger version holds, then CR is also $\kappa$-reducible and, therefore, hyperdecidable.
\end{abstract}

\section{INTRODUCTION}

Word problems (or rather the decidability thereof) have long played an important role in various branches of Mathematics. In some contexts a property can be associated with a decision problem by which the problem can be reduced in the sense that if it has a solution in an enlarged universe then it has a solution in the restricted universe. The first author and Steinberg [7] (see also [8]) have shown that two such properties on recursively enumerable pseudovarieties $\mathrm{V}_{1}, \ldots, \mathrm{V}_{n}$ of finite semigroups (which are then said to be tame) together are strong enough to guarantee decidability of their semidirect product $\mathrm{V}_{1} * \cdots * \mathrm{~V}_{n}$, whereas in general such a semidirect product is not decidable if the factors are only assumed to be decidable [17]. Although examples of tame pseudovarieties do not abound in the literature, and it may be quite to hard to establish tameness, it appears natural to conjecture that they are quite common $[3,17]$. The most famous example of a tame pseudovariety is the pseudovariety $\mathbf{G}$ of all finite groups, a result due to Ash [12].

Specifically, the properties in question involve an enlarged algebraic signature (made up of implicit operations) which have a natural interpretation on (pro)finite semigroups and concern the solution of the word problem for relatively free objects with respect to

Received 19th December, 2000

The first author gratefully acknowledges support by FCT through the Centro de Matemática da Universidade do Porto, by the project Praxis/2/2.1/MAT/63/94, and by the Department of Mathematics of the University of Tasmania.

Copyright Clearance Centre, Inc. Serial-fee code: 0004-9727/01 \$A2.00+0.00. 
this signature, as well as a reduction property for inevitability of graph labellings. A generalised version of such word problems is the pseudoidentity problem, that is to say, to obtain criteria for equality over the given pseudovariety of two arbitrary implicit operations. While there are too many implicit operations to even consider this question from an algorithmic point of view, when attention is restricted to specific implicit signatures, a measure of the effectiveness of such criteria is whether they yield true algorithmic solutions of the corresponding word problems.

In this paper, we consider a specific example, namely the pseudovariety $\mathbf{C R}$ of all finite completely regular semigroups. Completely regular semigroups are unions of groups. Such semigroups have received considerable attention in recent years in the realm of the algebraic theory of semigroups. They are in a sense close enough to groups to allow the development of a theory in which many problems are reduced to problems in group theory, although often in rather nontrivial ways. This is the case in particular, for the word problem for the free completely regular semigroup (as a unary semigroup) which was obtained by Kadourek and Polák [14]. It is one of the ingredients in establishing the criterion for equality in the free profinite completely regular semigroup presented in Section 4. The other ingredients are basically the study of combinatorial properties of the characteristic sequence introduced by Kadourek and Polák.

Rather than showing that $\mathbf{C R}$ is a tame pseudovariety, we show that this property would follow from a strengthened version of Ash's inevitability theorem [12, Theorem 2.1] in which collapse of generators is admitted. In view of the interest Ash's theorem has attracted over the past decade and its connections with other areas of Mathematics (see $[5,6,18])$, it appears to be worthwhile proposing this as an open problem.

\section{Preliminaries}

The reader is referred to [1] for general background and motivation on the theory of finite semigroups. See also [10] for an emphasis on the profinite aspects of the theory.

In the three subsections of this section, we either recall results from other papers or introduce preliminary results and notation which will play a role in the development of the main results.

We denote by $\mathbf{S l}$ the pseudovariety of all finite semilattices. For a pseudovariety $\mathbf{H}$ of groups, let $\overline{\mathrm{H}}$ denote the pseudovariety consisting of all finite semigroups all of whose subgroups lie in $\mathbf{H}$.

2.1. Content, 0, 1 Functions Let $S$ be an $A$-generated semigroup, that is, a function $\iota: A \rightarrow S$ is given whose image generates the semigroup $S$. We say that $S$ has content function $c$ if $c: S^{1} \rightarrow \mathcal{P}(A)$ is a monoid homomorphism into the semilattice of all subsets of $A$ under union such that $c(\iota a)=\{a\}$ for every $a \in A$. Thus, there is at most one content function on the $A$-generated semigroup $S$. We shall always denote content functions, irrespective of the semigroup, by $c$. Assuming $S$ has a content function, we 
define for each $s \in S, O(s)$ to be the set of all $s_{1} \in S^{1}$ such that there is a factorisation $s=s_{1} a s_{2}$ with $c\left(s_{1}\right) \neq c(s)=c\left(s_{1}\right) \cup\{a\} ;$ the set of all such $a \in A$ is denoted by $\overline{0}(s)$. The sets $1(s)$ and $\overline{1}(s)$ are defined dually. While the sets $0(s)$ and $\overline{0}(s)$ are always nonempty they need not be singletons. The semigroup $S$ is said to have 0 (respectively $\overline{0}, 1, \overline{1}$ ) function if $0(s)$ (respectively $\overline{0}(s), 1(s), \overline{1}(s)$ ) is a singleton for every $s \in S$. The free semigroup $A^{+}$and the free band on $A$ both have $0, \overline{0}, 1, \overline{1}$ functions.

From [9, Section 3], it follows that, for every finite $A$-generated semigroup $S$, there is an $A$-generated finite semigroup $T$ with content, $0, \overline{0}, 1$, and $\overline{1}$ functions for which there is a homomorphism $T \rightarrow S$ which respects the choice of generators (generally speaking, a semigroup $T$ for which there exists such a homomorphism is called an expansion of $S$ ). The idea is to first find an (easy) expansion of $S$ which has a content function and then apply the Birget expansion to that to obtain the other functions. If $S$ lies in a pseudovariety which contains $\mathbf{S l}$ and is closed under Birget expansion, then it follows that $S$ has an expansion in the same pseudovariety which has content, $0, \overline{0}, 1, \overline{1}$ functions. This holds for instance for the pseudovarieties CR [16] and $\overline{\mathbf{H}}[\mathbf{1 3}$, XII. (9.4)] for every pseudovariety $\mathbf{H}$ of groups and therefore also for $\mathbf{C R} \cap \overline{\mathbf{H}}$. By a standard argument, the free pro-V semigroup on a set $A, \bar{\Omega}_{A} \mathrm{~V}$, has content $0,1, \overline{0}, \overline{1}$ functions provided $\mathrm{V}$ contains $\mathrm{Sl}$ and is closed under Birget expansion. For later reference, we summarise the relevant results as follows.

PROPOS I TION 2.1. Every finite A-generated semigroup has a finite $A$-generated expansion which has content, $0, \overline{0}, 1$, and $\overline{1}$ functions.

PRoposition 2.2. For every pseudovariety $\mathbf{H}$ of groups, $\bar{\Omega}_{A}(\mathrm{CR} \cap \overline{\mathbf{H}})$ has content; $0, \overline{0}, 1$, and $\overline{1}$ functions.

2.2. Graphs AND SEMigroupoids By a graph $\Gamma$ we mean a set partitioned into a set $V(\Gamma)$, of vertices, and $E(\Gamma)$, of edges, together with two unary operations $\alpha, \omega: E(\Gamma) \rightarrow$ $V(\Gamma)$ giving the initial and end vertices for each edge. A semigroupoid is a graph endowed with a partial associative operation on edges such that, for two edges $s$ and $t, s t$ is defined if and only if $\omega s=\alpha t$, and then $\alpha(s t)=\alpha s$ and $\omega(s t)=\omega t$.

Graph and semigroupoid homomorphisms are defined as functions sending vertices to vertices and edges to edges and respecting the operations involved in each case. A subgraph (respectively a subsemigroupoid) of a graph (respectively semigroupoid) $\Gamma$ is a structure of the same kind on a subset $\Delta$ of $\Gamma$ such that the inclusion $\Delta \hookrightarrow \Gamma$ is a homomorphism. Products and coproducts are also defined in the natural way.

A semigroupoid homomorphism $S \rightarrow T$ is said to be faithful if it is injective on each set of edges $\left\{s \in E(S): \alpha s=v_{1}, \omega s=v_{2}\right\}$ with $v_{1}, v_{2} \in V(S)$, and it is said to be a quotient homomorphism if it is surjective and it is injective on $V(S)$. We say that a semigroupoid $S$ divides a semigroupoid $T$ if there exists a semigroupoid $U$, a faithful homomorphism $U \rightarrow T$ and a quotient homomorphism $U \rightarrow S$. A pseudovariety of semigroupoids is a class of finite semigroupoids which contains the 1-vertex 1-edge 
semigroupoid and is closed under taking divisors and finitary products and coproducts. The pseudovariety of all finite semigroupoids is denoted Sd.

A relational morphism $\mu: S \rightarrow T$ of structures of the same kind (semigroups, monoids, graphs, semigroupoids) is a relation $\mu \subseteq S \times T$ with domain $S$ which is a substructure of $S \times T$.

For a graph $\Gamma$, denote by $\Gamma^{+}$the free semigroupoid on $\Gamma$, which has the same vertex set as $\Gamma$ and whose edges are the nontrivial paths of $\Gamma$. If $W$ is a pseudovariety of semigroupoids and $\Gamma$ is a finite graph, then we say that a subset $L$ of $E\left(\Gamma^{+}\right)$is $\mathbf{W}$ recognisable if there is a semigroupoid homomorphism $\varphi: \Gamma^{+} \rightarrow S$ into a member of W such that $\varphi^{-1} \varphi L=L$.

For a semigroupoid $S$, we define its consolidation $S_{\mathrm{Cd}}$ to be the semigroup $E(S)$ under multiplication of edges, with a zero adjoined if $S$ has edges which cannot be multiplied, such products being then defined to be zero. The set of edges with beginning and end at a given vertex $v$, if nonempty, is a semigroup under edge multiplication and it is called the local semigroup at $v$. A semigroup $S$ is viewed as a 1-vertex semigroupoid, namely as the local semigroup at the single vertex. Note that, for a semigroupoid $S$, the natural homomorphism $S \rightarrow S_{\mathrm{Cd}}$ is a faithful homomorphism.

For a pseudovariety $\mathrm{V}$ of semigroups, its global is the pseudovariety of semigroupoids $g \mathbf{V}$ generated by the members of $\mathbf{V}$ viewed as semigroupoids.

2.3. F-Recognisability and F-Rationality Throughout this subsection, let $B$ denote a profinite graph, meaning a projective limit of finite graphs. For a pseudovariety $\mathbf{W}$ of semigroupoids, the free pro-W semigroupoid over $B$ will be denoted by $\bar{\Omega}_{B} \mathrm{~W}$. This semigroupoid is characterised by the following universal property: there is a canonical continuous graph homomorphism $\iota: B \rightarrow \bar{\Omega}_{B} \mathrm{~W}$ and, if $\varphi: B \rightarrow S$ is a continuous graph homomorphism into a semigroupoid from $W$, then there is a unique continuous semigroupoid homomorphism $\hat{\varphi}: \bar{\Omega}_{B} \mathrm{~W} \rightarrow S$ such that $\hat{\varphi} \iota=\varphi$. Such free semigroupoids are easily constructed, just as in the case $B$ is finite and W is pseudovariety of semigroups, as the projective limit of all continuous graph homomorphisms $B \rightarrow S$ whose image generates $S$ (see $[10,11]$ ). In the case where $\mathrm{W}$ is globally non trivial (that is; it includes a semigroupoid with distinct coterminal edges), then since $B$ is profinite, the canonical mapping $\iota$ is injective and so we think of $B$ as a subgraph of $\bar{\Omega}_{B} \mathrm{~W}$.

We extend the work of the first author for the case of finite sets $B$, pseudovarieties of semigroups and topological characterisations of recognisable sets (see [1, Section 3.6]) to show how the topology of $\bar{\Omega}_{B} \mathrm{~W}$ is intimately related with certain subsets of the free semigroupoid $B^{+}$over the graph $B$. Actually, taking into account the intended applications in this paper and elsewhere, we introduce the further restriction that $B$ be a clopen subset of $\bar{\Omega}_{A} \mathrm{~V}$ for a pseudovariety $\mathrm{V}$ of semigroups and a finite set $A$. Since elements of $B$ can then be viewed as both elements of $\bar{\Omega}_{A} \mathrm{~V}$ and of $\bar{\Omega}_{B} \mathrm{~W}$, where convenient we distinguish the latter by writing $\langle b\rangle$ for $b \in B$. More generally, for $P \subseteq B$, 
we write $\langle P\rangle$ to denote $\{\langle b\rangle: b \in P\}$.

Let $B_{0}$ denote the intersection of $B$ with the free semigroup $A^{+}$. Note that the metric structure of $B_{0}$ determined by the natural (ultra)metric on $A^{+}$associated with the pseudovariety $\mathrm{V}$ (see [1, Section 3.4]) is such that $B$ is its completion. We further assume that $B_{0}$ is a subgraph of $B$, that is, $\alpha\left(B_{0}\right) \cup \omega\left(B_{0}\right) \subseteq B_{0}$. Then $V\left(B_{0}\right)$ and $E\left(B_{0}\right)$ are V-recognisable subsets of $A^{+}$(see [1, Theorem 3.6.1]).

We say that a subset $L$ of $E\left(\left\langle B_{0}\right\rangle^{+}\right)$is $(\mathrm{V}, \mathrm{W})$-recognisable if there is a homomorphism $\varphi:\left\langle B_{0}\right\rangle^{+} \rightarrow S$ into $S \in \mathrm{W}$ such that the restriction $\left.\varphi\right|_{B_{0}}$ is uniformly continuous and $L=\varphi^{-1} \varphi L$. We also say that $L \subseteq E\left(\left\langle B_{0}\right\rangle^{+}\right)$is $(\mathrm{V}, \mathrm{W})$-rational if there are a finite graph $C$, a W-recognisable subset $K$ of $E\left(C^{+}\right)$, and a graph homomorphism $\lambda: B_{0} \rightarrow C$ such that each $\lambda^{-1} c(c \in C)$ is a V-recognisable subset of $A^{+}$, and, for the natural extension $\mu:\left\langle B_{0}\right\rangle^{+} \rightarrow C^{+}$to a semigroupoid homomorphism, $L=\mu^{-1} \mathrm{~K}$. In informal terms, $L$ is obtained from a $\mathbf{W}$-recognisable language by substituting each edge by a $\mathrm{V}$ recognisable subset of $\left\langle B_{0}\right\rangle$. In case $\mathbf{V}=\mathrm{S}$ is the pseudovariety of all finite semigroups and $\mathbf{W}=\mathrm{Sd}$ is the pseudovariety of all finite semigroupoids, then we say that a subset of $\left\langle B_{0}\right\rangle^{+}$is $F$-recognisable (respectively $F$-rational) if it is (S,Sd)-recognisable (respectively (S,Sd)-rational).

Proposition 2.3. A subset $L$ of $E\left(\left\langle B_{0}\right\rangle^{+}\right)$is $(\mathrm{V}, \mathrm{W})$-recognisable if and only if it is $(\mathrm{V}, \mathbf{W})$-rational.

Proof: Suppose first that $L$ is $(\mathrm{V}, \mathrm{W})$-recognisable and let $\varphi: B_{0}^{+} \rightarrow S$ be as in the definition of $(\mathrm{V}, \mathrm{W})$-recognisable set. Since the sets $V\left(B_{0}\right)$ and $E\left(B_{0}\right)$ are $\mathrm{V}$-recognisable and $\left.\varphi\right|_{B_{0}}$ is uniformly continuous, there is a congruence of finite index $\theta$ on $A^{+}$saturating $V\left(B_{0}\right)$ and $E\left(B_{0}\right)$, whose restriction to $B_{0}$ is contained in the congruence $\left.\operatorname{ker} \varphi\right|_{B_{0}}$, and such that $A^{+} / \theta \in \mathrm{V}$. In particular, the classes of $\left.\operatorname{ker} \varphi\right|_{B_{0}}$ are $\mathrm{V}$-recognisable subsets of $A^{+}$. Let $C$ be the quotient set $B_{0} /\left.\operatorname{ker} \varphi\right|_{B_{0}}$, which in fact is a graph in a natural way since $\left.\varphi\right|_{B_{0}}: B_{0} \rightarrow S$ is a graph homomorphism. Let $\lambda: B_{0} \rightarrow C$ be the natural projection. Then $\lambda^{-1} c$ is a $\mathrm{V}$-recognisable subset of $A^{+}$for every $c \in C$. The mapping $\left.\varphi\right|_{B_{0}}: B_{0} \rightarrow S$ induces an (injective) graph homomorphism $C \rightarrow S$ which extends uniquely to a semigroupoid homomorphism $\psi: C^{+} \rightarrow S$. The various mappings are depicted in the following commutative diagram:

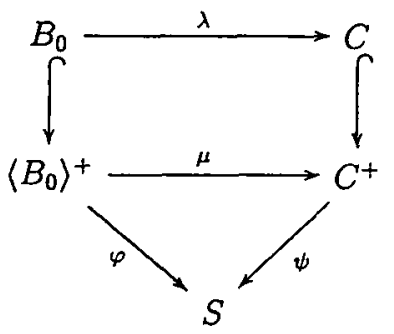

Let $K=\psi^{-1} \varphi L$ and take $\mu$ as in the definition of $(\mathrm{V}, \mathrm{W})$-rational set. Then, for 
$w_{1}, \ldots, w_{n} \in B_{0}$ such that $\left\langle w_{1}\right\rangle \cdots\left\langle w_{n}\right\rangle \in E\left(\left\langle B_{0}\right\rangle^{+}\right)$, we have the following chain of equivalences:

$$
\begin{aligned}
\left\langle w_{1}\right\rangle \cdots\left\langle w_{n}\right\rangle \in L & \Leftrightarrow\left\langle\varphi w_{1}\right) \cdots\left(\varphi w_{n}\right) \in \varphi L \\
& \quad \text { since } \varphi \text { is a homomorphism on }\left\langle B_{0}\right\rangle^{+} \text {whose kernel saturates } L \\
& \Leftrightarrow\left(\varphi w_{1}\right) \cdots\left(\varphi w_{n}\right) \in \psi K \\
& \quad \text { since } \psi K=\psi \psi^{-1} \varphi L=\varphi L \text { as } \varphi \text { and } \psi \text { have the same image } \\
& \Leftrightarrow\left(\psi \lambda w_{1}\right) \cdots\left(\psi \lambda w_{n}\right) \in \psi K \quad \text { since } \psi \lambda=\left.\varphi\right|_{B_{0}} \\
& \Leftrightarrow \psi\left(\left(\lambda w_{1}\right) \cdots\left(\lambda w_{n}\right)\right) \in \psi K \quad \text { since } \psi \text { is a homomorphism } \\
& \Leftrightarrow\left(\lambda w_{1}\right) \cdots\left(\lambda w_{n}\right) \in K \quad \text { since } \psi^{-1} \psi K=K \\
& \Leftrightarrow\left\langle w_{1}\right\rangle \cdots\left\langle w_{n}\right\rangle \in \mu^{-1} K .
\end{aligned}
$$

This shows that $L$ is $(\mathbf{V}, \mathbf{W})$-rational.

Conversely, assume that $L$ is $(\mathrm{V}, \mathbf{W})$-rational and let $C, K, \lambda, \mu$ be as in the definition of (V,W)-rational set. Let $\psi: C^{+} \rightarrow S$ be a homomorphism into a semigroupoid of $\mathbf{W}$ recognising $K$. Then $\varphi=\psi \mu:\left\langle B_{0}\right\rangle^{+} \rightarrow S$ is a semigroupoid homomorphism and

$$
\varphi^{-1} \varphi L=\varphi^{-1} \varphi\left(\mu^{-1} \psi^{-1} \psi K\right)=\varphi^{-1} \varphi \varphi^{-1} \psi K=\varphi^{-1} \psi K=\mu^{-1} \psi^{-1} \psi K=L
$$

which shows that $L$ is $(\mathrm{V}, \mathbf{W})$-recognisable.

Note that, if $\mathbf{W}=g(\mathbf{W} \cap \mathbf{S})$, that is if $\mathbf{W}$ is the global of a pseudovariety of semigroups, then the semigroupoid $S$, and therefore also the graph $C$, in the above proof may be taken to have just one vertex.

In particular, we have the following special case.

Corollary 2.4. A subset $L$ of $E\left(\left\langle B_{0}\right\rangle^{+}\right)$is F-recognisable if and only if it is F-rational.

From the definition of $\bar{\Omega}_{B} \mathrm{~W}$ it follows that this compact zero-dimensional space has the initial topology for the homomorphisms $\varphi$ into semigroupoids from $W$ such that the restrictions $\left.\varphi\right|_{B}$ are continuous. In particular, the clopen subsets of the form $\varphi^{-1} Q$, where $\varphi: \bar{\Omega}_{B} \mathbf{W} \rightarrow S$ is a homomorphism into a semigroupoid from W and $Q \subseteq S$, form a basis of the topology of $\bar{\Omega}_{B} \mathbf{W}$, in which $V\left(\bar{\Omega}_{B} \mathbf{W}\right)$ and $E\left(\bar{\Omega}_{B} \mathbf{W}\right)$ are clopen subsets. We now express the edge part of this topology in terms of $(V, W)$-recognisable subsets of $E\left(\left\langle B_{0}\right\rangle^{+}\right)$.

\section{THEOREM 2.5 .}

(a) A closed subset $P$ of $E\left(\bar{\Omega}_{B} W\right)$ is clopen if and only if $P \cap E\left(\left\langle B_{0}\right\rangle^{+}\right)$is $(\mathrm{V}, \mathrm{W})$-recognisable and dense in $P$.

(b) A subset $L$ of $E\left(\left\langle B_{0}\right\rangle^{+}\right)$is $(\mathrm{V}, \mathrm{W})$-recognisable if and only if $L=P \cap$ $E\left(\left\langle B_{0}\right\rangle^{+}\right)$for some clopen subset $P$ of $E\left(\bar{\Omega}_{B} \mathrm{~W}\right)$. 
(c) A subset $P$ of $E\left(\bar{\Omega}_{B} \mathrm{~W}\right)$ is clopen if and only if it is the closure in $\bar{\Omega}_{B} \mathrm{~W}$ of some $(\mathrm{V}, \mathrm{W})$-recognisable subset of $E\left(\left\langle B_{0}\right\rangle^{+}\right)$.

Proof: Let $P$ be a clopen subset of $E\left(\bar{\Omega}_{B} \mathrm{~W}\right)$. By the remarks preceding the statement of the theorem, since $P$ is compact, there is a homomorphism $\varphi: \bar{\Omega}_{B} \mathbf{W} \rightarrow S$ with $S \in \mathrm{W}$ and $Q \subseteq S$ such that $P=\varphi^{-1} Q$ and $\left.\varphi\right|_{B}$ is continuous. Then $P \cap$ $E\left(\left\langle B_{0}\right\rangle^{+}\right)=\left(\left.\varphi\right|_{\left\langle B_{0}\right\rangle^{+}}\right)^{-1} Q$ is a $(\mathbf{V}, \mathbf{W})$-recognisable subset by the definition of such sets. This proves half of (b). On the other hand, it is easy to verify that $E\left(\left\langle B_{0}\right\rangle^{+}\right)$is a dense subset of $E\left(\bar{\Omega}_{B} \mathrm{~W}\right)$. Since $P$ is open, it follows that $P \cap E\left(\left\langle B_{0}\right\rangle^{+}\right)$is dense in $P$, thus proving half of (a).

For the converse in (b), assume that $L$ is a $(\mathbf{V}, \mathbf{W})$-recognisable subset of $E\left(\left\langle B_{0}\right\rangle^{+}\right)$. Then there is a homomorphism $\varphi:\left\langle B_{0}\right\rangle^{+} \rightarrow S$ into a semigroupoid $S$ from W and a subset $Q$ of $S$ such that $L=\varphi^{-1} Q$ and $\left.\varphi\right|_{B_{0}}$ is uniformly continuous. By the universal property of $\bar{\Omega}_{B} \mathrm{~W}$ there is a unique extension of $\varphi$ to a continuous homomorphism $\hat{\varphi}$ : $\bar{\Omega}_{B} \mathrm{~W} \rightarrow S$. Let $P=\widehat{\varphi}^{-1} Q$. Then $P$ is a clopen subset of $E\left(\bar{\Omega}_{B} \mathrm{~W}\right)$ and $P \cap E\left(\left\langle B_{0}\right\rangle^{+}\right)=$ $\left(\left.\widehat{\varphi}\right|_{\left(B_{0}\right)^{+}}\right)^{-1} Q=L$.

For the converse in (a), suppose that $L=P \cap E\left(\left\langle B_{0}\right\rangle^{+}\right)$is (V,W)-recognisable and dense in the closed set $P$. By (b) there is some clopen subset $Q$ of $E\left(\bar{\Omega}_{B} \mathrm{~W}\right)$ such that $Q \cap E\left(\left\langle B_{0}\right\rangle^{+}\right)=L$. Since $E\left(\left\langle B_{0}\right\rangle^{+}\right)$is dense in $E\left(\bar{\Omega}_{B} \mathrm{~W}\right)$ and $Q$ is open, $L$ must be dense in $Q$. Since $L$ is also dense in $P$ and $P$ is closed, it follows that $P=Q$ and so $P$ is clopen.

Part (c) follows from (a) and (b).

In particular, continuity of functions to and from $\bar{\Omega}_{B} \mathrm{~W}$ may be expressed combinatorially in terms of $(\mathrm{V}, \mathrm{W})$-recognisable languages. For later usage, we formulate the following special cases. Recall that a function between topological spaces is said to be open if it maps open sets to open sets.

Note that Theorem 2.5 (c) generalises the well known description of V-recognisable languages [1].

Corollary 2.6 .

(a) A function $\varphi: A^{+} \rightarrow E\left(\left\langle B_{0}\right\rangle^{+}\right)$is uniformly continuous with respect to $\mathrm{V}$, $\mathrm{W}$ (that is, it extends to a unique continuous function $\bar{\Omega}_{A} \mathrm{~V} \rightarrow E\left(\bar{\Omega}_{B} \mathrm{~W}\right)$ ) if and only if, for every $(\mathrm{V}, \mathrm{W})$-recognisable $L \subseteq E\left(\left\langle B_{0}\right\rangle^{+}\right), \varphi^{-1} L$ is $\mathrm{V}$ recognisable.

(b) A uniformly continuous function $\varphi: A^{+} \rightarrow E\left(\left\langle B_{0}\right\rangle^{+}\right)$with respect to $\mathrm{V}$, $\mathrm{W}$, is such that its unique continuous extension $\bar{\Omega}_{A} \mathrm{~V} \rightarrow E\left(\bar{\Omega}_{B} \mathrm{~W}\right)$ is open if and only if, for every $\mathrm{V}$-recognisable subset $L$ of $\Omega_{A} \mathrm{~V}, \varphi L$ is $(\mathrm{V}, \mathrm{W})$ recognisable.

The theory of F-rational languages can thus be developed as a generalisation of the theory of rational languages of a finitely generated free semigroup. We shall use freely 
other results, such as the closure of the class of F-rational languages under the finitary Boolean operations and left and right quotients. The following property will also be used without further proof.

LEMmA 2.7. There is an algorithm to compute, for a given F-rational language $L \subseteq E\left(\left\langle B_{0}\right\rangle^{+}\right)$and a given homomorphism $\psi:\left\langle B_{0}\right\rangle^{+} \rightarrow S$ into a finite semigroupoid $S$, the set $\psi L$.

\section{THE CHARACTERISTIC SEQUENCE}

The characteristic sequence of a unary word (meaning a term in a free unary semigroup) was introduced by Kadourek and Polák [14] in connection with their solution of the word problem for free completely regular semigroups. A similar construction was introduced by the second author in an earlier independent solution of the same problem [19]. We are presently interested only in semigroup words, not involving the extra unary operation, although the extension we shall consider of the characteristic sequence will also extend, in a sense which we shall not analyze here, the Kadourek and Polák definition since the unary operation can be viewed as the local inversion $x \mapsto x^{\omega-1}$.

For the remainder of this paper, we let $A$ denote a finite set and for $w \in A^{+}$we let $|w|$ denote the length of $w$ (not to be confused with the notation $|X|$ for the cardinality of a set $X)$. We define a map $\chi: A^{+} \rightarrow\left\langle A^{+}\right\rangle^{+}$as follows: for $w \in A^{+}$with $|c(w)|>1$, let $\chi(w)=\left\langle w_{1}\right\rangle \cdots\left\langle w_{r}\right\rangle$ if $w=u_{i} w_{i} v_{i},\left|c\left(w_{i}\right)\right|=|c(w)|-1$, the last letter of $u_{i}$ (in case $u_{i} \neq 1$ ) and the first letter of $v_{i}$ (in case $v_{i} \neq 1$ ) do not belong to $c\left(w_{i}\right), 0 \leqslant\left|u_{1}\right|<\cdots<\left|u_{r}\right| \leqslant|w|$, and there are no other such maximal factors $w_{i}$ of $w$ whose content misses just one letter of the content of $w$. Note that, for $w \in A^{+}, \chi(w)$ is always a word of length at least 2, the first and the last letter being, respectively $0(w)$ and $1(w)$. For $w \in A^{+}$, if $w=u a v$ with $a=\overline{0}(w)$ and $u=0(w)$, then we write $0^{\prime}(w)=a v$. The function $I^{\prime}$ is defined dually.

The inverse image under $\chi$ stands for the reconstruction of a word from its characteristic sequence. This reconstruction is essentially obvious and is given in the following lemma (see [15, Result 5.3]).

LEMMA 3.1. Let $v=\left\langle w_{1}\right\rangle \cdots\left\langle w_{n}\right\rangle \in\left\langle A^{+}\right\rangle^{+}$. Then $v$ belongs to the image of $\chi$ if and only if $n \geqslant 2$ and the following conditions hold:

$$
\begin{aligned}
& \left|c\left(w_{1}\right)\right|=\cdots=\left|c\left(w_{n}\right)\right| \\
& c\left(w_{i}\right) \neq c\left(w_{i+1}\right) \text { and } 1\left(w_{i}\right)=0\left(w_{i+1}\right) \text { for } i=1, \ldots, n-1 .
\end{aligned}
$$

Moreover, then the only $w \in A^{+}$such that $\chi(w)=v$ is given by $w=w_{1} \cdot 0^{\prime}\left(w_{2}\right) \cdots 0^{\prime}\left(w_{n}\right)$. In particular, the function $\chi$ is injective.

For $X \subseteq A$, denote by $[X]$ the set of all elements of $A^{+}$of content $X$. See $[\mathbf{1}$, Section 8.1] for an extension of the content function to implicit operations on pseudovarieties of semigroups containing the pseudovariety $\mathrm{Sl}$ of all finite semilattices. 
3.1. EXTENSION To $\bar{\Omega}_{A} \mathrm{~S}$ Let $\mathrm{V}$ be a pseudovariety of semigroups containing $\mathrm{Sl}, P \subseteq$ $\bar{\Omega}_{A} \mathrm{~V}$, and let $X$ be a subset of $A$ with at least 2 elements. We define a graph $\partial_{X} P$ on the set of all factors $v \in\left(\bar{\Omega}_{A} \mathrm{~V}\right)^{1}$ of elements of $P$ such that $|X \backslash c(v)| \in\{1,2\}$ by letting the vertices be those $v$ such that $|X \backslash c(v)|=2$ and the edges be the remaining elements of $\partial_{X} P$, the adjacency functions being $\alpha=0$ and $\omega=1$. Note in particular that $V\left(\partial_{X} A^{+}\right)$and $E\left(\partial_{X} A^{+}\right)$are rational languages of $A^{+}$which are in fact Sl-recognisable.

The significance of the graph $\partial_{X} A^{+}$comes from Lemma 3.1. Indeed, the characteristic sequences of words of content $X$ can be viewed as paths in the graph, although not all paths fulfil the conditions of the lemma. More precisely, we have the following result.

Proposition 3.2. The set $\chi[X]$ is an F-rational subset of the free semigroupoid $\left\langle\partial_{X} A^{+}\right\rangle^{+}$.

Proof: Define on $\partial_{X} A^{+}$a relation $\sim$ as follows:

for all $v_{1}, v_{2} \in V\left(\partial_{X} A^{+}\right), v_{1} \sim v_{2}$;

for $w_{1}, w_{2} \in E\left(\partial_{X} A^{+}\right), w_{1} \sim w_{2}$ if $c\left(w_{1}\right)=c\left(w_{2}\right)$.

The quotient set $C=\partial_{X} A^{+} / \sim$ is then a finite set and it is in fact a 1-vertex graph in a natural way such that the canonical mapping $\mu: \partial_{X} A^{+} \rightarrow C$ is a graph homomorphism. Moreover, the $\sim$-classes are Sl-recognisable subsets of $A^{+}$.

For each edge $w / \sim$ of $C$, we have an associated "content" $c(w / \sim)=c(w)$. For each edge $w=x_{1} \cdots x_{n}$ of $C^{+}$, with the $x_{i} \in E(C)$, define

$$
\chi_{c}(w)=\left\{\left(c\left(x_{i}\right), c\left(x_{i+1}\right)\right): i=1, \ldots, n-1\right\} .
$$

Define on $E\left(C^{+}\right)$a relation $\simeq$ by letting, for coterminal $w_{1}, w_{2} \in E\left(C^{+}\right), w_{1} \simeq w_{2}$ if $w_{1}$ and $w_{2}$ start with the same edges, end with the same edges, and $\chi_{c}\left(w_{1}\right)=\chi_{c}\left(w_{2}\right)$. Then $S=E\left(C^{+}\right) / \simeq$ is a finite set and a finite semigroupoid in a natural way so that $\psi: C^{+} \rightarrow S$ is a homomorphism.

To complete the proof, in view of Lemma 3.1 and Proposition 2.3, it suffices to observe that the set $\chi[X]$ is precisely the set of all paths $w \in E\left(\left\langle\partial_{X} A^{+}\right\rangle^{+}\right)$such that $\chi_{c}(w)$ contains no pair $(c(x), c(y))$ with $c(x)=c(y)$.

Let $A_{2}$ denote the multiplicative semigroup consisting of the matrices

$$
\left(\begin{array}{ll}
1 & 0 \\
0 & 0
\end{array}\right),\left(\begin{array}{ll}
0 & 1 \\
0 & 0
\end{array}\right),\left(\begin{array}{ll}
1 & 0 \\
1 & 0
\end{array}\right),\left(\begin{array}{ll}
0 & 0 \\
1 & 1
\end{array}\right),\left(\begin{array}{ll}
0 & 0 \\
0 & 0
\end{array}\right) .
$$

Then it is well known that the semigroupoid $S$ in the proof of Proposition 3.2 belongs to the global of the pseudovariety $\mathrm{V}\left(A_{2}\right)$ of all so-called 1 -testable finite semigroups (see $[13,4])$. Hence, in the terminology of subsection 2.3 , we have the following result.

COROLlary 3.3. The set $\chi[X]$ is an $\left(\mathrm{Sl}, g \mathrm{~V}\left(A_{2}\right)\right)$-recognisable subset of $E\left(\left\langle\partial_{X} \cdot A^{+}\right\rangle^{+}\right)$. 
We wish to extend the function $\chi$ to continuous functions $\overline{[X]} \rightarrow \bar{\Omega}_{\partial_{X} \bar{\Omega}_{A} \mathrm{~S}} \mathrm{Sd}$ and $\overline{[X]} \rightarrow \bar{\Omega}_{\partial_{X} \bar{\Omega}_{A} \mathrm{CR}} \mathrm{G}$, where $\overline{[X]}$ is the closure of $[X]$ in $\bar{\Omega}_{A} \mathrm{~S}$ and $\bar{\Omega}_{A} \mathrm{CR}$ respectively. By Corollary 2.6, the existence of such functions means the uniform continuity of $\chi$ with respect to various uniform structures and translates combinatorially in terms of the inverse image under $\chi$ of certain F-rational languages being rational languages of a certain type.

LEMMA 3.4. Let $L$ be a rational language of $A^{+}$and suppose that it is recognised by a homomorphism $\varphi: A^{+} \rightarrow S$ onto a finite semigroup $S$ possessing content and 0 functions. Then $O(L)$ and $O^{\prime}(L)$ are both recognised by $\varphi$ and $L$ can be partitioned into a finite number of rational languages $L_{i}$ such that $L_{i}=0\left(L_{i}\right) 0^{\prime}\left(L_{i}\right)$ with each of the languages $0\left(L_{i}\right)$ and $0^{\prime}\left(L_{i}\right)$ recognised by $\varphi$.

PRoOF: Note that $\varphi 0=0 \varphi$. On the other hand, for $s \in \varphi L$ and $s=s_{1} s_{2}$, we have $\left(\varphi^{-1} s_{1}\right)\left(\varphi^{-1} s_{2}\right) \subseteq \varphi^{-1} s \subseteq L$. Taking $s_{1}=0(s)$, we conclude that $0(L)=\bigcup\left\{\varphi^{-1} s_{1}: s_{1} \in\right.$ $0(\varphi L)\}$ and $0^{\prime}(L)=\bigcup\left\{\varphi^{-1} s_{2}: s \in \varphi L, s=0(s) \cdot s_{2}\right\}$. Finally, for each $s \in \varphi L$ and each $s_{2}$ such that $s=0(s) \cdot s_{2}$, let $L_{s, s_{2}}=\left(\varphi^{-1} 0(s)\right)\left(\varphi^{-1} s_{2}\right)$. Then the rational languages $L_{s, s_{2}}$ partition $L$ into finitely many parts and $0\left(L_{s, s_{2}}\right)=\varphi^{-1} 0(s)$, and $0^{\prime}\left(L_{s, s_{2}}\right)=\varphi^{-1} s_{2}$.

By Kleene's Theorem, we obtain the following result.

Corollary 3.5. Let $L$ be a rational language of $A^{+}$. Then $0(L)$ and $0^{\prime}(L)$ are also rational languages and there is a partition of $L$ into finitely many rational languages $L_{i}$ such that $L_{i}=0\left(L_{i}\right) 0^{\prime}\left(L_{i}\right)$.

We can now show by Corollary 2.6 that $\left.\chi\right|_{[x]}$ is uniformly continuous.

Proposition 3.6. For every F-rational subset $L$ of $E\left(\left\langle\partial_{X} A^{+}\right\rangle^{+}\right)$, the language $\chi^{-1} L \subseteq A^{+}$is rational.

PROOF: In this proof we restrict our attention to F-rational subsets of $L$ that are components of $L$ under various decompositions. Since, say by Theorem 2.5 , the intersection of two F-rational subsets of $E\left(\left\langle\partial_{X} A^{+}\right\rangle^{+}\right)$is again F-rational, by Proposition 3.2 we may assume that $L \subseteq \chi[X]$. By Lemma 3.1, it follows that $\chi^{-1} L$ consists of all words of the form $w_{1} \cdot 0^{\prime}\left(w_{2}\right) \cdots 0^{\prime}\left(w_{n}\right)$ such that $\left\langle w_{1}\right\rangle\left\langle w_{2}\right\rangle \cdots\left\langle w_{n}\right\rangle \in L$. Let $C, K, \lambda, \mu$ describe $L$ as in the definition of $(\mathbf{S}, \mathbf{S d})$-rational set. Since $\mathbf{S d}=g \mathbf{S}$, we may assume that $C$ is a 1-vertex graph.

By partitioning $K$ into a finite number of rational languages, we may assume that all words in $K$ start with the same letter $x_{0} \in E(C)$. By introducing a new letter in $C$, if necessary, we may assume that $x_{0}$ only occurs as the first letter of words in $K$. By assumption, each language $\lambda^{-1} x \subseteq A^{+}(x \in E(C))$ is rational and so, by Corollary 3.5 , so is $0^{\prime}\left(\lambda^{-1} x\right)$. Hence $\chi^{-1} L$ is the language obtained from $K$ by substituting $x_{0}$ by $\lambda^{-1} x_{0}$ and the remaining $x \in E(C)$ by $0^{\prime}\left(\lambda^{-1} x\right)$. Now, it is well known and easy to show that, if $K$ is a rational language over a finite alphabet, then by replacing all letters by rational languages of $A^{+}$, we obtain a rational language of $A^{+}$. Hence $\chi^{-1} L \subseteq A^{+}$is rational. 
Let $\varphi: A^{+} \rightarrow S$ be a homomorphism onto a finite semigroup with content, 0 , and 1 functions. We associate with $S$ and a subset $X$ of $A$ with at least 2 elements a semigroupoid $\vec{S}_{X}$ defined as follows. The vertices of $\vec{S}_{X}$ are the elements of $S^{1}$ whose content is contained in $X$ but miss precisely two elements of $X$. The edges of $\vec{S}_{X}$ are just the elements of $S$ whose content is contained in $X$ and miss at most one element of $X$. The adjacency functions are given by $\alpha(s)=0(0(s))$ and $\omega(s)=1(1(s))$ for edges $s$ of content $X$ and by $\alpha(s)=0(s)$ and $\omega(s)=1(s)$ for edges whose content misses a letter of $X$. The product $s_{1} \diamond s_{2}$ of consecutive edges $s_{1}, s_{2} \in E\left(\vec{S}_{X}\right)$ is obtained by taking $s_{1} s_{2}^{\prime}$ where $s_{2}^{\prime}$ is any element of $S$ such that $s_{2}=\alpha\left(s_{2}\right) s_{2}^{\prime}$. If $s_{2}^{\prime \prime}$ is another element of $S$ such that $s_{2}=\alpha\left(s_{2}\right) s_{2}^{\prime \prime}$, then, taking an arbitrary $t \in S$ such that $s_{1}=t \omega\left(s_{1}\right)$, we conclude that

$$
s_{1} s_{2}^{\prime}=t \omega\left(s_{1}\right) s_{2}^{\prime}=t \alpha\left(s_{2}\right) s_{2}^{\prime}=t \alpha\left(s_{2}\right) s_{2}^{\prime \prime}=t \omega\left(s_{1}\right) s_{2}^{\prime \prime}=s_{1} s_{2}^{\prime \prime}
$$

Similarly, we can show that $\omega\left(s_{1} s_{2}^{\prime}\right)=\omega\left(s_{2}\right)$, while obviously $\alpha\left(s_{1} s_{2}^{\prime}\right)=\alpha\left(s_{1}\right)$. Hence the product $\diamond$ is well defined and it is easily verified that it is associative, thus showing that $\vec{S}_{X}$ is indeed a semigroupoid.

Consider the function $\partial_{X} A^{+} \rightarrow \vec{S}_{X}$ defined by sending each $w \in \partial_{X} A^{+}$to $\varphi w$ and note that it is a graph homomorphism since $S$ has content and 0 and 1 functions. It is also obviously uniformly continuous with respect to the metric structure of $\partial_{X} A^{+}$defined by the natural metric on $A^{+}$since it is a restriction of the uniformly continuous mapping $\varphi$. This graph homomorphism extends uniquely to a semigroupoid homomorphism $\varphi_{S, X}$ : $\left\langle\partial_{X} A^{+}\right\rangle^{+} \rightarrow \vec{S}_{X}$.

PROPOSITION 3.7. The function $\left.\chi\right|_{[X]}$ sends rational languages of $A^{+}$to $F$ rational languages of $E\left(\left\langle\partial_{X} A^{+}\right\rangle^{+}\right)$.

Proof: Let $L$ be a rational language of $A^{+}$contained in $[X]$.

Let $\varphi: A^{+} \rightarrow S$ be a homomorphism onto a finite semigroup $S$ which recognises $L$. Note that $L$ is also recognised by any semigroup divisible by $S$. By Proposition 2.1, we may thus assume that $S$ possesses content, 0 , and 1 functions.

Consider the semigroupoid $\vec{S}_{X}$ constructed above and the associated homomorphism $\varphi_{S, X}:\left\langle\partial_{X} A^{+}\right\rangle^{+} \rightarrow \vec{S}_{X}$. We claim that $\chi L=\varphi_{S_{,} X}^{-1} \varphi L \cap \chi[X]$, which will establish the desired result in view of Proposition 3.2.

If $w \in[X]$ and $\chi w=\left\langle w_{1}\right\rangle \cdots\left\langle w_{n}\right\rangle$, then the path $\left(\left\langle w_{1}\right\rangle, \ldots,\left\langle w_{n}\right\rangle\right)$ in the graph $\partial_{X} A^{+}$(that is, the corresponding edge in the free semigroupoid $\left\langle\partial_{X} A^{+}\right\rangle^{+}$) is mapped under $\varphi$ to the edge product $\varphi w_{1} \diamond \cdots \diamond \varphi w_{n}$ which is easily recognised to be precisely $\varphi w$. Hence $w \in L$ if and only if $\chi w \in \varphi_{S, X}^{-1} \varphi L$ and this proves the claim.

In view of Corollary 2.6, we have thus established the following result.

THEOREM 3.8. For each subset $X$ with at least two elements of a finite alphabet $A$, the characteristic sequence mapping $\chi$ extends uniquely to a continuous mapping

$$
\chi^{X}: \overline{[X]} \rightarrow \bar{\Omega}_{\partial_{X} \bar{\Omega}_{A} S} \mathrm{Sd}
$$


where $\left[\overline{X]}\right.$ denotes the closure of $[X]$ in $\bar{\Omega}_{A} \mathrm{~S}$. Moreover, the mapping $\chi^{X}$ is open.

Let $V, V_{1}$, and $V_{2}$ be pseudovarieties of semigroups such that $V_{1} \supseteq V_{2} \supseteq S l$ and let $\mathbf{W}, \mathbf{W}_{1}$, and $\mathbf{W}_{2}$ be pseudovarieties of semigroupoids such that $\mathbf{W}_{1} \supseteq \mathbf{W}_{2}$. For each subset $X$ of $A$ with at least two elements, the canonical projection $p_{\mathrm{V}_{1}, \mathrm{~V}_{2}}: \bar{\Omega}_{A} \mathrm{~V}_{1} \rightarrow \bar{\Omega}_{A} \mathrm{~V}_{2}$ induces a continuous graph homomorphism $\partial_{X} \bar{\Omega}_{A} V_{1} \rightarrow \partial_{X} \bar{\Omega}_{A} V_{2}$ which extends to a unique continuous semigroupoid homomorphism $q_{\mathbf{V}_{1}, \mathbf{V}_{2}}^{\mathbf{W}}: \bar{\Omega}_{\partial_{X} \bar{\Omega}_{A} \mathbf{V}_{1}} \mathbf{W} \rightarrow \bar{\Omega}_{\partial_{X} \bar{\Omega}_{A} \mathbf{V}_{2}} \mathbf{W}$. We thus have the following commutative diagram of continuous semigroupoid homomorphisms where, for a profinite graph $\Gamma$, the canonical projection $\bar{\Omega}_{\Gamma} \mathbf{W}_{1} \rightarrow \bar{\Omega}_{\Gamma} \mathbf{W}_{2}$ is also denoted by $p_{\mathbf{w}_{1}, \mathbf{w}_{2}}$ :

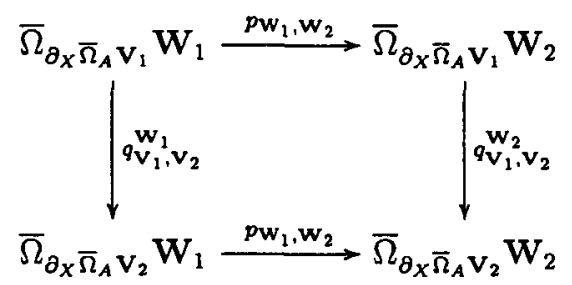

In particular, we may define a (continuous) function $\chi_{\mathrm{V}, \mathrm{W}}^{X}: \overline{[X]} \subseteq \bar{\Omega}_{A} \mathrm{~S} \rightarrow \bar{\Omega}_{\partial_{X} \bar{\Omega}_{A} \mathrm{~V}} \mathrm{~W}$ by taking

$$
\chi_{\mathbf{V}, \mathbf{w}}^{X}=q_{\mathbf{S}, \mathbf{v}}^{\mathbf{w}} \circ p_{\mathbf{S d}, \mathbf{W}} \circ \chi^{X} .
$$

A question of interest in view of the applications in this paper is under what conditions the function $\chi_{\mathbf{V}, \mathbf{W}}^{X}$ factorises through the canonical projection $p_{\mathbf{S}, \mathbf{V}}$. Although some examples are presented in the next subsection, we have no general characterisation of the pairs $\mathrm{V}, \mathrm{W}$ for which there is such a factorisation.

3.2. Extension to $\bar{\Omega}_{A}$ CR From [15, Theorem 3.6], one immediately deduces the following result.

THEOREM 3.9. For any pseudovariety $\mathbf{H}$ of groups, if $u, v \in A^{+}$, then the identity $u=v$ holds in $\mathbf{C R} \cap \overline{\mathbf{H}}$ if and only if all of the following conditions hold:

1. $c(u)=c(v)$;

2. $\mathbf{C R} \cap \overline{\mathbf{H}} \models 0(u)=0(v)$;

3. $\mathbf{C R} \cap \overline{\mathbf{H}}=1(u)=1(v)$;

4. either $|c(u)|=1$ and $\mathbf{H} \vDash u=v$, or $|c(u)|>1$ and $\mathbf{H} \vDash \chi_{\mathbf{C R} \cap \overline{\mathbf{H}}, \mathbf{S d}}^{X} u=$ $\chi_{\mathrm{CR} \cap \overline{\mathrm{H}}, \mathrm{Sd}}^{X} v$, where $X=c(u)$.

In the following we also use systematically the following result which follows from [15, Proposition 2.2].

PROPOSITION 3.10. If $\mathrm{H}$ is a finitely generated pseudovariety of groups, then the pseudovariety $\mathbf{C R} \cap \overline{\mathbf{H}}$ is locally finite.

We consider now the composite $\left.q_{\mathrm{S}, \mathrm{CR} \cap \overline{\mathrm{H}}}^{g \mathrm{H}} \circ p_{\mathrm{Sd}, g \mathrm{H}} \circ \chi\right|_{[X]}$. 
PROPOSITION 3.11. For $|X| \geqslant 2$, the mapping $\left.\chi\right|_{[X]}:[X] \rightarrow\left\langle\partial_{X} A^{+}\right\rangle^{+}$is uniformly continuous with respect to the canonical pro-CR $\cap \overline{\mathbf{H}}$ metric of $A^{+}$and the canonical pro- $(\mathrm{CR} \cap \overline{\mathrm{H}}, g \mathrm{H})$ uniformity of $\left\langle\partial_{X} A^{+}\right\rangle^{+}$.

Proof: Consider a Cauchy sequence $\left(w_{n}\right)_{n}$ in $[X]$ (with respect to the canonical pro-CR $\cap \overline{\mathbf{H}}$ metric of $\left.A^{+}\right)$. Let $G \in \mathbf{H}$ and let $\varphi:\left\langle\partial_{X} A^{+}\right\rangle^{+} \rightarrow G$ be a semigroupoid homomorphism (where $G$ is viewed as a one-vertex semigroupoid with the vertex missing) whose restriction to the graph $\partial_{X} A^{+}$is continuous with respect to the canonical pro$\mathrm{CR} \cap \overline{\mathbf{H}}$ metric of $A^{+}$. We must show that there is some $n_{0}$ such that $n \geqslant n_{0}$ implies $\varphi \chi w_{n}=\varphi \chi w_{n_{0}}$.

Since the restriction of $\varphi$ to $\partial_{X} A^{+}$is continuous, it induces a rational partition of $A^{+}$. Since $\partial_{X} A^{+}$is itself a recognisable subset of $A^{+}$, there is some congruence of finite index $\theta$ on $A^{+}$which saturates every class in the partition and also the set $\partial_{X} A^{+}$. Let $\mathrm{W}=$ $\mathrm{V}(G) \vee\left(\mathrm{V}\left(A^{+} / \theta\right) \cap \mathrm{G}\right)$ where $\mathrm{V}(G)$ and $\mathrm{V}\left(A^{+} / \theta\right)$ are the pseudovarieties generated by the indicated semigroups. Let $S=\bar{\Omega}_{A} \mathrm{U}$ where $\mathrm{U}=\mathrm{CR} \cap \overline{\mathrm{W}} ;$ so $A^{+} / \theta \in \mathrm{U}$. Note that $S$ is finite by Proposition 3.10. If $\psi:\left\langle\partial_{X} A^{+}\right\rangle^{+} \rightarrow\left\langle\partial_{X} \bar{\Omega}_{A} U\right\rangle^{+}$denotes the semigroupoid homomorphism induced by the canonical projection $A^{+} \rightarrow \bar{\Omega}_{A} \mathrm{U}$, then $\operatorname{ker} \psi \subseteq \operatorname{ker} \varphi$ as we now show. Note that $\psi$ is a literal homomorphism between free semigroupoids, in the sense that it sends edges of the generating graph $\partial_{X} A^{+}$of the first free semigroupoid to edges of the generating graph $\partial_{X} \bar{\Omega}_{A} \mathrm{U}$ of the second free semigroupoid. Hence it suffices to verify that $\left.\operatorname{ker} \psi\right|_{\partial_{X} A^{+}} \subseteq \operatorname{ker} \varphi$. Indeed, since $A^{+} / \theta \in U$, we have $\left.\operatorname{ker} \psi\right|_{\partial_{X} A^{+}} \subseteq \theta \subseteq \operatorname{ker} \varphi$.

Now, from the inclusion $\operatorname{ker} \psi \subseteq \operatorname{ker} \varphi$, it follows that there exists a semigroupoid homomorphism $\delta:\left\langle\partial_{X} \bar{\Omega}_{A} \mathrm{U}\right\rangle^{+} \rightarrow G$ such that $\delta \psi=\varphi$. The situation is depicted in the following commutative diagram.

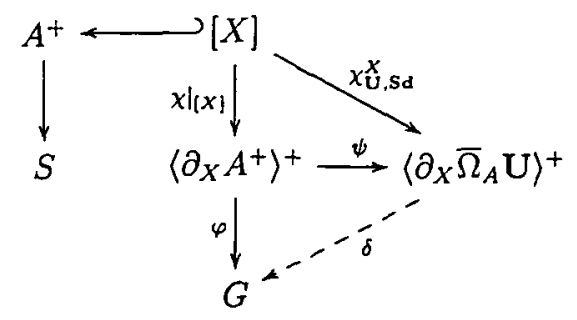

Since $\left(w_{n}\right)_{n}$ is a Cauchy sequence in $A^{+}$, there is $n_{0}$ such that, for all $n \geqslant n_{0}, S \models w_{n}=$ $w_{n_{0}}$. Since in fact $S=\bar{\Omega}_{A} \mathrm{U}$, we also have $\mathrm{U} \vDash w_{n}=w_{n_{0}}$ for all $n \geqslant n_{0}$. By Theorem 3.9, $\mathrm{W} \vDash \chi_{\mathrm{U}, \mathrm{Sd}}^{X} w_{n}=\chi_{\mathrm{U}, \mathrm{Sd}}^{X} w_{n_{0}}$ for all $n \geqslant n_{0}$. Hence, for $n \geqslant n_{0}$, the following equalities hold

$$
\varphi \chi w_{n}=\delta \chi_{\mathbf{U}, \mathbf{S d}}^{X} w_{n}=\delta \chi_{\mathbf{U}, \mathbf{S d}}^{X} w_{n_{0}}=\varphi \chi w_{n_{0}}
$$

where the middle equality follows from the fact that $G \in \mathrm{W}$.

COROLlary 3.12. For each pseudovariety $\mathrm{H}$ of groups and each $X \subseteq A$ with 
at least two elements, the mapping $\chi$ induces a unique continuous function

$$
\widehat{\chi}_{\mathbf{C R} \cap \overline{\mathrm{H}}, g \mathbf{H}}^{X}: \overline{[X]} \rightarrow \bar{\Omega}_{\partial_{X} \bar{\Omega}_{\Lambda} \mathbf{C R} \cap \overline{\mathrm{H}}} g \mathrm{H},
$$

where $\overline{[X]}$ denotes the closure of $[X]$ in $\bar{\Omega}_{A} \mathrm{CR} \cap \overline{\mathrm{H}}$.

The following commutative diagram may help to keep track of the various functions involved.

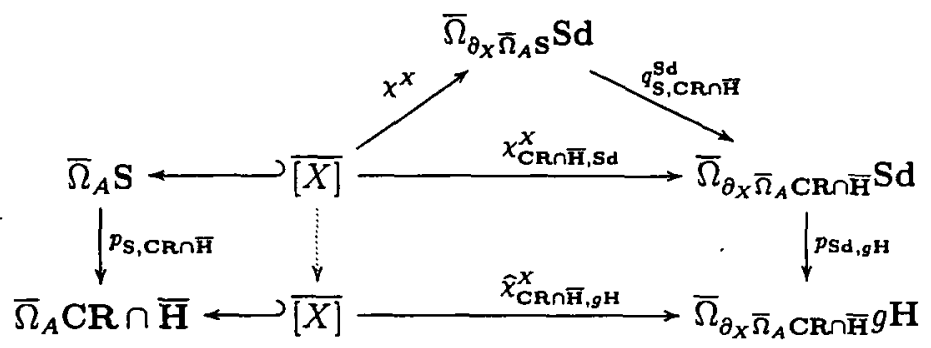

\section{THE WORD PROBLEM FOR $\bar{\Omega}_{A}$ CR}

It is now rather easy to give a "solution" of the word problem for the profinite semigroups $\bar{\Omega}_{A} \mathrm{CR}$. More generally, we have the following result.

THEOREM 4.1. For any pseudovariety $\mathbf{H}$ of groups and any $u, v \in \bar{\Omega}_{A} \mathbf{S}$, the pseudoidentity $u=v$ holds in $\mathrm{CR} \cap \overline{\mathrm{H}}$ if and only if each of the following conditions holds:

1. $c(u)=c(v)$;

2. $\mathbf{C R} \cap \overline{\mathbf{H}} \models 0(u)=0(v)$;

3. $\mathbf{C R} \cap \overline{\mathbf{H}} \models 1(u)=1(v)$;

4. either $|c(u)|=1$ and $\mathbf{H} \vDash u=v$, or $|c(u)|>1$ and $\mathbf{H} \models \chi_{\mathbf{C R} \cap \overline{\mathbf{H}}, \mathbf{S d}}^{X} u=$ $\chi_{\mathrm{CR} \cap \overline{\mathbf{H}}, \mathrm{Sd}}^{X} v$, where $X=c(u)$.

Proof: Suppose first that the pseudoidentity $u=v$ holds in $\mathbf{C R} \cap \overline{\mathbf{H}}$. Since $\mathbf{S l} \subseteq \mathbf{C R} \cap \overline{\mathbf{H}}$, the profinite free objects over the pseudovariety $\mathbf{C R} \cap \overline{\mathbf{H}}$ have a content function and so condition (i) certainly holds. Conditions (ii) and (iii) follow from the fact that $\bar{\Omega}_{A} \mathrm{CR} \cap \overline{\mathrm{H}}$ possesses 0 and 1 functions as guaranteed by Proposition 2.2. Finally, for condition (iv), the case $|X|=1$ is obvious, so we assume $|X|>1$. Then the condition $\mathbf{H} \models \chi_{\mathbf{C R} \cap \overline{\mathbf{H}}, \mathbf{S d}}^{X} u=\chi_{\mathbf{C R} \cap \overline{\mathbf{H}}, \mathbf{S d}}^{X} v$ is equivalent to $p_{\mathbf{S d}, g \mathbf{H}} \chi_{\mathbf{C R} \cap \overline{\mathbf{H}}, \mathbf{S d}}^{X} u=p_{\mathbf{S d}, g \mathbf{H}} \chi_{\mathbf{C R} \cap \overline{\mathbf{H}}, \mathbf{S d}}^{X} v$ which in view of the commutativity of the diagram (1), means that $\widehat{\chi}_{\mathrm{CR} \cap \overline{\mathbf{H}}, g \mathrm{H}}^{X} p_{\mathrm{S}, \mathrm{CR} \cap \overline{\mathrm{H}}} u=$ $\widehat{\chi}_{\mathrm{CR} \cap \overline{\mathbf{H}}, g \mathbf{H}}^{X} p_{\mathrm{S}, \mathrm{CR} \cap \overline{\mathrm{H}}^{v}}$. The necessity of the condition then follows from the assumption that $p_{\mathrm{S}, \mathrm{CR} \cap \overline{\mathrm{H}}} u=p_{\mathrm{S}, \mathrm{CR} \cap \overline{\mathrm{H}}} v$.

Conversely, suppose that the conditions (i)-(iv) hold. Let $X=c(u)$. If $|X|=1$, then clearly $\mathbf{C R} \cap \overline{\mathbf{H}} \vDash u=v$ since the one-generated members of $\mathbf{C R} \cap \overline{\mathbf{H}}$ are the 
cyclic groups in $\mathbf{H}$. So, assume that $|X|>1$. It suffices to show that $\mathbf{C R} \cap \overline{\mathbf{W}} \vDash u=v$ for every finitely generated subpseudovariety $\mathrm{W}$ of $\mathrm{H}$. For such a $\mathrm{W}$ we deduce from Proposition 3.10 that there are words $u^{\prime}, v^{\prime} \in A^{+}$such that $c\left(u^{\prime}\right)=X=c\left(v^{\prime}\right), \mathbf{C R} \cap \overline{\mathrm{W}}$ satisfies $u^{\prime}=u, v^{\prime}=v, 0\left(u^{\prime}\right)=0(u), 0\left(v^{\prime}\right)=0(v), 1\left(u^{\prime}\right)=1(u), 1\left(v^{\prime}\right)=1(v)$, and $\mathrm{W}$ satisfies the pseudoidentities $\chi_{\mathrm{CR} \cap \overline{\mathrm{H}}, \mathbf{S d}}^{X} u^{\prime}=\chi_{\mathrm{CR} \cap \overline{\mathrm{H}}, \mathrm{Sd}}^{X} u$ and $\chi_{\mathrm{CR} \cap \overline{\mathrm{H}}, \mathbf{S d}}^{X} v^{\prime}=\chi_{\mathrm{CR} \cap \overline{\mathrm{H}}, \mathrm{Sd}}^{X} v$. From (ii) and (iii), it follows that $\mathbf{C R} \cap \overline{\mathrm{W}}$ satisfies $0\left(u^{\prime}\right)=0\left(v^{\prime}\right)$ and $1\left(u^{\prime}\right)=1\left(v^{\prime}\right)$. From (iv), we deduce that $\mathrm{W} \vDash \chi_{\mathrm{CR} \cap \overline{\mathrm{W}}, \mathbf{S d}}^{X} u^{\prime}=\chi_{\mathrm{CR} \cap \overline{\mathrm{w}}, \mathbf{S d}}^{X} v^{\prime}$. Hence, by Theorem 3.9, we conclude that $\mathrm{CR} \cap \overline{\mathrm{W}}$ satisfies the identity $u^{\prime}=v^{\prime}$ and so also the pseudoidentity $u=v$ in view of the choice of $u^{\prime}, v^{\prime}$.

REMARK. It is legitimate to ask in what sense Theorem 4.1 gives a solution of the word problem for $\bar{\Omega}_{A}(\mathbf{C R} \cap \overline{\mathrm{H}})$. If $H$ is a locally finite pseudovariety of groups with computable finitely generated free objects, then Theorem 4.1 contains a solution of the word problem for $\bar{\Omega}_{A}(C R \cap \overline{\mathrm{H}})$ but it is one which reduces to Theorem 3.9. In the general case, say if $u, v \in \Omega_{A}^{\sigma} \mathrm{S}$ with $\sigma$ an implicit signature (see Section 5 for details) such that the functions $c, 0,1$, and $\chi_{\mathbf{C R n} \overline{\mathbf{H}}, \mathbf{S d}}^{X}$ are computable on $\Omega_{A}^{\sigma} \mathbf{S}$ and take values within the same signature, and the word problem is solvable for $\Omega_{B}^{\sigma} \mathrm{H}$, then from Theorem 4.1 it follows that the word problem for $\Omega_{A}^{\sigma}(\mathrm{CR} \cap \overline{\mathrm{H}})$ is solvable in the usual sense. Thus, although strictly speaking Theorem 4.1 does not solve any word problem in an algorithmic sense, it provides a theoretical characterisation of equality over $\mathbf{C R} \cap \overline{\mathbf{H}}$ which yields the solution of word problems for many members of $\mathbf{C R} \cap \overline{\mathbf{H}}$.

\section{OPEN PROBLEM: AN EXTENSION OF ASH'S RESULTS}

Throughout this section, we fix a mapping $\varrho: A \rightarrow B$ between finite sets. We denote by $\mathrm{V}$ an arbitrary pseudovariety of finite semigroups.

By a $\varrho$-relational morphism we mean a relational morphism $\mu: S \rightarrow T$ where $S$ is an $A$-generated semigroup, $T$ is a $B$-generated semigroup and $(a, \varrho a) \in \mu$ for all $a \in A$.

A labelling of a graph $\Gamma$ by a semigroup $S$ is a function $\gamma: \Gamma \rightarrow S^{1}$. The labelling $\gamma$ is said to be consistent if, for every edge $x \in E(\Gamma)$, the equality $(\gamma \alpha x)(\gamma x)=\gamma \omega x$ holds.

Two labellings $\gamma$ and $\delta$ of a graph $\Gamma$ respectively by semigroups $S$ and $T$ are said to be $\mu$-related under a relational morphism $\mu: S \rightarrow T$ if, under the canonical extension $\mu: S^{1} \rightarrow T^{1}$ for every $x \in \Gamma,(\gamma x, \delta x) \in \mu$.

We say that a labelling $\gamma$ of the graph $\Gamma$ by the $A$-generated semigroup $S$ is inevitable with respect to a $\varrho$-relational morphism $\mu: S \rightarrow T$ if there is a consistent labelling $\delta$ of $\Gamma$ by $T$ which is $\mu$-related with $\gamma$. We also say that $\gamma$ is $(\mathrm{V}, \varrho)$-inevitable if it is inevitable with respect to every $\varrho$-relational morphism $\mu: S \rightarrow T$ with $T \in \mathrm{V}$. In case $B=A$ and $\varrho$ is the identity function, we then say that $\gamma$ is $\mathrm{V}$-inevitable. The pseudovariety $\mathrm{V}$ is said to be hyperdecidable if there is an algorithm to decide whether a labelling of a finite graph by a finite semigroup is $\mathbf{V}$-inevitable. 
The following result extends [3, Proposition 3]. The proof is obtained by a straightforward adaptation of the proof of the original result and is therefore omitted.

Proposition 5.1. A labelling $\gamma$ of a finite graph $\Gamma$ by a finite $A$-generated semigroup is $(\mathrm{V}, \varrho)$-inevitable if and only if it is inevitable with respect to the canonical $\varrho$-relational morphism $\nu=\widehat{\varrho} \circ \varphi^{-1}$ where $\varphi: \bar{\Omega}_{A} \mathrm{~S} \rightarrow S$ is the homomorphism induced by the choice of generators and $\widehat{\varrho}: \bar{\Omega}_{A} \mathrm{~S} \rightarrow \bar{\Omega}_{B} \mathrm{~V}$ is the only continuous homomorphism whose restriction to $A$ is equal to $\varrho$.

By an implicit signature we mean, as in [7], a set $\sigma$ of implicit operations on finite semigroups which contains the basic semigroup multiplication. Profinite semigroups are then viewed naturally as $\sigma$-semigroups. In particular, for a pseudovariety $\mathbf{V}$ of semigroups, the $\sigma$-subsemigroup of $\bar{\Omega}_{A} \mathrm{~V}$ generated by $A$ is denoted by $\Omega_{A}^{\sigma} \mathrm{V}$. This is easily seen to be precisely the free object freely generated by the set $A$ in the variety of $\sigma$ semigroups generated by $\mathrm{V}$.

The most commonly used implicit signature consists of the basic semigroup multiplication together with the $\omega-1$ power which associates to each element $s$ of a finite semigroup $S$ the inverse $s^{\omega-1}$ of $s^{\omega+1}=s s^{\omega}$ in the subsemigroup generated by $s$, where $s^{\omega}$ denotes the only idempotent power of $s$. This signature is denoted by $\kappa$. In particular, $\Omega_{A}^{\kappa} S$ is the free object on the set $A$ in the unary semigroup variety for which $\omega-1$ is the unary operation.

Given an $A$-generated finite semigroup $S$ and a pseudovariety $\mathrm{V}$ of semigroups, the canonical $(\sigma, \varrho)$-relational morphism $\mu_{\mathrm{V}}^{\sigma}: S \rightarrow \Omega_{B}^{\sigma} \mathrm{V}$ is the composite $\widehat{\varrho} \circ \varphi^{-1}$ of the inverse $\varphi^{-1}$ of the unique homomorphism of $\sigma$-semigroups $\varphi: \Omega_{A}^{\sigma} \mathrm{S} \rightarrow S$, determined by the choice of generators, with the natural homomorphism of $\sigma$-semigroups $\widehat{\varrho}: \Omega_{A}^{\sigma} \mathrm{S} \rightarrow \Omega_{B}^{\sigma} \mathrm{V}$ determined by the mapping $\varrho$. We say that the pseudovariety $\mathrm{V}$ is $(\sigma, \varrho)$-reducible if a labelling of a finite graph by a finite $A$-generated semigroup $S$ is $(\mathrm{V}, \varrho)$-inevitable if and only if it is inevitable with respect to the canonical $(\sigma, \varrho)$-relational morphism $\mu_{\mathbf{V}}^{\sigma}: S \rightarrow \Omega_{B}^{\sigma} \mathbf{V}$. In case $\varrho$ is the identity function, we say that $\mathbf{V}$ is $\sigma$-reducible for $A$-generated semigroups if it is $(\sigma, \varrho)$-reducible. Finally, we say that $\mathrm{V}$ is $\sigma$-reducible if, for every finite set $A, \mathrm{~V}$ is $\sigma$-reducible for $A$-generated semigroups.

Now, Ash's inevitability theorem may be phrased as stating that $\mathbf{G}$ is $\kappa$-reducible (see $[7,8]$ ). In the next section, we show that $\kappa$-reducibility of $\mathbf{C R}$ follows from a stronger property which we now state as an open problem.

PROBLEM 5.2. Is it true that, for every (onto) mapping $\varrho: A \rightarrow B$ between finite sets, $\mathbf{G}$ is $(\kappa, \varrho)$-reducible?

While we have no specific evidence that the answer should be affirmative, the extension of Ash's results which it would provide does not appear to be very significant. Yet, in trying to extend Ash's arguments to this situation, one quickly finds that the apparently harmless collapse of generators produced by $\varrho$ does not allow Ash's proof to carry through 
in a straightforward manner. On the other hand, the compactness argument that led to Proposition 5.1 does not require much change to handle the collapse in generators. So, at least it appears that to conjecture an affirmative answer to the problem is reasonable. The real motivation comes from Theorem 6.4.

\section{ON $\kappa$-REDUCIBILITY OF CR}

In this section we shall see that if Problem 5.2 has an affirmative answer then the pseudovariety $\mathbf{C R}$ is $\kappa$-tame (that is, $\mathbf{C R}$ is $\kappa$-reducible).

Let $A$ be a non-empty set and $S$ be a finite $A$-generated semigroup that has content, 0 and 1 functions defined on it. Denote by $\varphi: \bar{\Omega}_{A} \mathrm{~S} \rightarrow S$ the natural homomorphism that extends the identity map on $A$. Our aim is to show that, given a finite graph $\Gamma$, with $k$ vertices, and a labelling $\gamma: \Gamma \rightarrow S^{1}$, we can decide whether there exists a labelling $\delta: \Gamma \rightarrow\left(\bar{\Omega}_{A} \mathrm{~S}\right)^{1}$ such that $\varphi \delta=\gamma$ and $p_{\mathrm{CR}} \delta$ is consistent. Here $p_{\mathrm{CR}}: \bar{\Omega}_{A} \mathrm{~S} \rightarrow \bar{\Omega}_{A} \mathrm{CR}$ is the canonical projection. In other words we wish to show that we can decide whether $\gamma$ is CR-inevitable. Following [7] we shall aim at the more refined property of $\kappa$-reducibility, namely the following property: $\gamma$ is CR-inevitable if and only if there is a labelling $\delta$ as above, but which takes its values in $\left(\Omega_{A}^{\kappa} S\right)^{1}$. The converse is immediate.

Note that, if the labelling $\gamma$ as above can be lifted to a labelling $\delta$ over $\Omega_{A}^{\kappa} \mathrm{S}$ such that $p_{\mathbf{C R}} \delta$ is consistent, then one may effectively construct such a labelling. Indeed, we may recursively enumerate the candidate labellings over the recursively enumerable set $\Omega_{A}^{\kappa} \mathrm{S}$. For each candidate $\delta$, we may compute $\varphi \delta$ and check whether it is equal to $\gamma$. We may also compute the labelling $p_{\mathbf{C R}} \delta$ to test whether it is consistent using the solution of the word problem for $\mathbf{C R}$.

We shall show that there exists a finite computable subset $P$ of $\left(\Omega_{A}^{\kappa} S\right)^{1}$ such that for every finite graph $\Delta$ of at most $m$ vertices and every labelling $\tau: \Delta \rightarrow S^{1}, \tau$ is CR-inevitable only if there is a labelling $\pi: \Delta \rightarrow P$ such that $\varphi \pi=\tau$ and $p_{\mathbf{C R}} \pi$ is consistent. Observe that if two coterminal edges of $\Delta$ are labelled with the same element of $S^{1}$ then they may also be labelled with the same element of $\Omega_{A}^{\kappa} \mathrm{S}$. So we may assume $\Delta$ has at most $|S|+1$ distinct coterminal edges. Therefore we need consider only finitely many graphs with at most $m$ vertices; of course there are only finitely many $S$-labellings of these. So if $\mathbf{C R}$ is $\kappa$-reducible, then there must exist such a computable set $P$.

In order to show that $\mathrm{CR}$ is $\kappa$-reducible we shall make use of Theorem 4.1. Condition 4 of the theorem involves testing the function $\chi_{\mathbf{C R}, \mathbf{S d}}^{B}$, where $B$ has at least two elements; this is obtained by composing $\chi^{B}$ with a projection of $\bar{\Omega}_{\partial_{B} \bar{\Omega}_{B} \mathrm{~S}} \mathrm{Sd}$. Recall by Theorem 3.8 that $\chi^{B}: \overline{[B]} \rightarrow \bar{\Omega}_{\partial_{B} \bar{\Omega}_{B} \mathrm{~S}} \mathrm{Sd}$ is a continuous map on the closure of $[B]=\left\{w \in B^{+}: c(w)=B\right\}$ that extends the function $\chi$. A modification of the semigroupoid $\vec{S}_{B}$ of Section 3.1 will play an important role in the application of Theorem 4.1 ; in particular we make use of its consolidation monoid $\left(\vec{S}_{B}\right)_{\mathrm{Cd}}^{1}$ (see Section 2.2). This monoid can be defined in algebraic terms as follows. 
Let $S_{B}$ be the $B$-generated subsemigroup of $S^{1}$ and consider the subsets

$$
Z=\left\{w \in S_{B}:|B \backslash c(w)|=1\right\}, \quad T=\left\{w \in S_{B}:|B \backslash c(w)| \leqslant 1\right\} \cup\{1\} .
$$

For $w \in T \backslash\{1\}$ define

$$
\hat{\mathbf{1}}(w)=\left\{\begin{array}{cc}
1(1(w)) & \text { if } c(w)=B \\
1(w) & \text { if } c(w) \neq B
\end{array}\right.
$$

and define $\widehat{0}(w)$ dually. The set $Z$ generates the o-monoid $\left(T^{0}, 0\right)$ for $S$ with binary operation defined for $u, v \neq 1$ by

$$
u \diamond v=\left\{\begin{array}{lll}
u v^{\prime} & \text { if } \hat{1}(u)=\widehat{0}(v) & \text { where } v=\hat{0}(v) v^{\prime} \\
0 & \text { if } \hat{1}(u) \neq \hat{0}(v) & \text { or } u=0 \text { or } v=0
\end{array}\right.
$$

and 1 is the identity element.

We shall later obtain a graph from $\Gamma$ labelled by $\left(\bar{\Omega}_{\partial_{B}} \bar{\Omega}_{B} \mathbf{S} S\right)_{\text {Cd; }}^{1}$ this is a monoid in which we again let $\diamond$ denote the binary operation. It will be convenient to identify the partial semigroup $E\left(\bar{\Omega}_{\partial_{B} \bar{\Omega}_{B} S} \mathrm{Sd}\right)$ with the partial semigroup $\left(\bar{\Omega}_{\partial_{B} \bar{\Omega}_{B} S} \mathrm{Sd}\right)_{\mathrm{Cd}} \backslash\{0\}$ and to assume it contains the range of $\chi^{B}$. Notice that $\left(\bar{\Omega}_{\partial_{B}} \bar{\Omega}_{B} S \mathbf{S d}\right)_{\mathrm{Cd}}^{1}$ is the closure of the monoid $\left(\left\langle\partial_{B} \bar{\Omega}_{B} S\right\rangle^{+}\right)_{C d}^{1}$ which is generated as a $\diamond$-monoid by

$$
Y=\left\{w \in \bar{\Omega}_{B} \mathbf{S}:|B \backslash c(w)|=1\right\} .
$$

Define $\psi:\left(\bar{\Omega}_{\theta_{B} \bar{\Omega}_{B} S} \mathbf{S d}\right)_{\text {Cd }}^{1} \rightarrow\left(T^{0}, \diamond\right)$ to be the unique homomorphism that extends the action of $\varphi$ on the generating set $Y$. Observe that if $w \in[B]$ then, by Lemma 3.1, $\varphi(w)=\psi \chi^{B}(w)$. Furthermore since $\varphi, \psi$, and $\chi^{B}$ are continuous functions then on $\overline{[B]}$, $\varphi=\psi \chi^{B}$.

By [2, Proposition 2.3] the set $\left(\bar{\Omega}_{\partial_{B} \bar{\Omega}_{B} S} S d\right)_{C d} \backslash\{0\}$ embeds as a partial subsemigroup in $\bar{\Omega}_{Y} S$ (by an injective partial homomorphism $\mu$, say). The canonical homomorphism $\lambda: \bar{\Omega}_{Y} \mathrm{~S} \rightarrow\left(\bar{\Omega}_{\partial_{B} \bar{\Omega}_{B} \mathrm{~S}} \mathrm{Sd}\right)_{\mathrm{Cd}}$ that extends the identity map on $Y$ is such that $\lambda \mu$ is the identity map on its domain. In effect, $\mu$ and $\lambda$ interchange the binary operations $\diamond$ and .

Let us return to the problem of the existence of the set $P$.

If the labelling $\tau$ of $\Delta$ is by elements from $\left\{a \in S^{1}:|c(a)| \leqslant 1\right\}$ then, since $S$ satisfies a periodic law $x^{p+q}=x^{p}$, it can be easily seen that it suffices that $P$ includes $\left\{a^{i}, a^{\omega+i}: a \in A, 1 \leqslant i \leqslant p+q\right\}$. We proceed by induction, with the following assumptions for some $n, 1<n \leqslant|A|$ and each subset $B \subseteq A$ such that $|B|=n$. Let $U=\{w \in$ $\left.\left(\bar{\Omega}_{B} \mathbf{S}\right)^{1}: c(w) \subsetneq B\right\}$.

There exists a finite subset $P^{\prime}$ of $U \cap\left(\Omega_{A}^{\kappa} S\right)^{1}$ and a map $\theta: U \rightarrow P^{\prime}$ such that

(i) $\theta^{2}=\theta, \theta 0=0 \theta, \theta 1=1 \theta$ and $\varphi \theta=\varphi$,

(ii) for each $u, v \in U$,

$$
p_{\mathrm{CR}}(u)=p_{\mathrm{CR}}(v) \Rightarrow p_{\mathrm{CR}} \theta(u)=p_{\mathrm{CR}} \theta(v) .
$$


(iii) for every finite graph $\Delta$ with at most $\max \{k, 3|S|\}$ vertices and each labelling $\tau: \Delta \rightarrow U$ such that $p_{\mathbf{C R}} \tau$ is consistent then $p_{\mathbf{C R}} \theta \tau$ is consistent.

(iv) for each $u \in P^{\prime}, x \in P^{\prime} \cap \varphi^{-1} \varphi 0(u) \cap p_{\mathrm{CR}}^{-1} p_{\mathrm{CR}} 0(u)$ and $y \in P^{\prime} \cap \varphi^{-1} \varphi 1(u) \cap$ $p_{\mathrm{CR}}^{-1} p_{\mathrm{CR}} 1(u)$ there exists $u_{x y} \in P^{\prime}$ such that $\varphi\left(u_{x y}\right)=\varphi(u), x=0\left(u_{x y}\right)$, $y=1\left(u_{x y}\right)$ and $u_{x y} \in p_{\mathrm{CR}}^{-1} p_{\mathrm{CR}}(u)$.

If $|B|=2$ then for any $w \in U, c(w) \leqslant 1$; it follows from the comments preceding the assumptions that $\theta$ can be chosen in this case to satisfy (i), (ii), (iii) and (iv). The aim is to show, given the assumptions, that $P^{\prime}$ can be extended to a finite subset of $\left(\Omega_{B}^{\kappa} S\right)^{1}$ and the domain of $\theta$ can be extended to $\left(\bar{\Omega}_{B} S\right)^{1}$ in such a way as to satisfy (i), (ii), (iii) and (iv).

Notice that the graph $\partial_{B} \bar{\Omega}_{B} \mathrm{~S}$ has a subgraph, denoted by $\theta \partial_{B} \bar{\Omega}_{B} \mathrm{~S}$, with edge set $\theta(Y)=\{\theta(w): w \in Y\}$. Let $\hat{\theta}:\left(\bar{\Omega}_{\partial_{B} \bar{\Omega}_{B} \mathbf{S}} \mathbf{S d}\right)_{\mathrm{Cd}}^{1} \rightarrow\left(\bar{\Omega}_{\theta \partial_{B} \bar{\Omega}_{B} \mathrm{~S}} \mathbf{S d}\right)_{\mathrm{Cd}}^{1}$ be the unique continuous homomorphism that extends $\left.\theta\right|_{Y}$. Of course $\left(\bar{\Omega}_{\theta \partial_{B}} \bar{\Omega}_{B} S \mathrm{Sd}\right)_{C d}^{1}$ embeds by $\mu$ as a partial subsemigroup in $\bar{\Omega}_{\theta(Y)}$ S. Since we are assuming that $\varphi \theta=\varphi$ on $Y$ then $\psi \widehat{\theta}=\psi$.

For any $r, s \in \bar{\Omega}_{B} S$ such that $c(r s) \neq B$ consider the graph $\Delta$ with $E(\Delta)=\{e\}$, $V(\Delta)=\{\alpha(e), \omega(e)\}$ and a labelling $\tau: \Delta \rightarrow \bar{\Omega}_{B} \mathrm{~S}$ given by $\tau \alpha(e)=r, \tau(e)=s$ and $\tau \omega(e)=r s$. Then $p_{\mathrm{CR}} \tau$ is consistent and hence, by the induction assumption (iii), $p_{\mathbf{C R}} \theta \tau$ is also consistent. Therefore $p_{\mathbf{C R}}(\theta(r) \theta(s))=p_{\mathbf{C R}} \theta(r s)$.

In the rest of the paper $A, S, \varphi$ and $\Gamma$ are as specified above and $B$ is a subset of $A$ with $|B|>1$. Let $\gamma: \Gamma \rightarrow S^{1}$ be a CR-inevitable labelling of $\Gamma$ by the $B$-generated submonoid of $S^{1}$ via the labelling $\delta: \Gamma \rightarrow\left(\bar{\Omega}_{B} \mathrm{~S}\right)^{1}$; that is, $\varphi \delta=\gamma$ and $p_{\mathbf{C R}} \delta$ is consistent. Note that $p_{\mathrm{CR}}$ and $\varphi$ preserve content and commute with the 0 and 1 functions. Since $p_{\mathrm{CR}} \delta$ is consistent then for any path in $\Gamma$ from $x$ to $y$ we have $c(\delta(x)) \subseteq c(\delta(y))$.

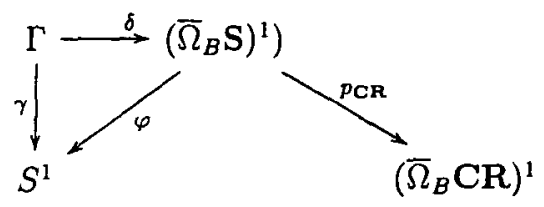

LEMMA 6.1. There is a labelling $\delta: \Gamma \rightarrow\left(\bar{\Omega}_{B} \mathrm{~S}\right)^{1}$ such that $\varphi \delta=\gamma, p_{\mathrm{CR}} \delta$ is consistent, and for each $z \in \mathrm{\Gamma}$,

(i) if $c \delta(z)=B$, then $\chi^{B} \delta(z)=\hat{\theta} \chi^{B} \delta(z)$ and, in particular, $0 \delta(z), 1 \delta(z) \in P^{\prime}$,

(ii) if $c \delta(z) \subsetneq B$ then $\delta(z) \in P^{\prime}$.

Proof: By Lemma 3.1 and induction assumption (i), if $w \in[B]$ then $\widehat{\theta} \chi^{B}(w)$ is in the range of $\chi$. Since $\widehat{\theta} \chi^{B}$ maps $\overline{[B]}$ continuously into $\bar{\Omega}_{\theta \partial_{B} \bar{\Omega}_{B} S} S d$ then the range of $\hat{\theta} \chi^{B}$ is within the range of $\chi^{B}$. So for $w \in \overline{[B]}$ we may select a $w_{\theta} \in \overline{[B]}$ such that $\widehat{\theta} \chi^{B}(w)=\chi^{B}\left(w_{\theta)}\right.$. 
Define a labelling $\delta^{\prime}: \Gamma \rightarrow\left(\bar{\Omega}_{B} \mathrm{~S}\right)^{1}$ by

$$
\delta^{\prime}(z)= \begin{cases}\theta \delta(z) & \text { if } c \delta(z) \neq B \\ \delta(z)_{\theta} & \text { if } c \delta(z)=B\end{cases}
$$

We shall see that the labelling $\delta^{\prime}$ satisfies the requirements of the Lemma.

Suppose $e \in E(\Gamma)$ and $c \delta \omega(e) \neq B$. Then $\varphi \delta^{\prime}(z)=\gamma(z)$ for $z \in\{\alpha(e), e, \omega(e)\}$ by the induction assumption (i), while by assumption (ii) and an observation following the induction assumptions $p_{\mathbf{C R}}\left(\delta^{\prime}(\alpha(e)) \delta^{\prime}(e)\right)=p_{\mathbf{C R}} \delta^{\prime} \omega(e)$.

Now suppose $e \in E(\Gamma)$ and $c \delta \omega(e)=B$. By Theorem 4.1, G $\models \chi_{\mathbf{C R}, \mathbf{S d}}^{B}(\delta \alpha(e) \delta(e))=$ $\chi_{\mathrm{CR}, \mathrm{Sd}}^{B} \delta \omega(e)$. If $c \delta \alpha(e)=B$ then $\chi^{B} \delta \alpha(e)=u \diamond x$ for some $u \in\left(\bar{\Omega}_{\partial_{B} \bar{\Omega}_{B} \mathrm{~S}} \mathrm{Sd}\right)_{\mathrm{Cd}}^{\mathbf{l}} \backslash\{0\}$ and $x=1 \delta \alpha(e)$. Dually if $c \delta(e)=B$ then $\chi^{B} \delta(e)=y \diamond v$ for some $v \in\left(\bar{\Omega}_{\partial_{B} \bar{\Omega}_{B} \mathrm{~S}} \mathrm{Sd}\right)_{\mathrm{Cd}}^{1} \backslash\{0\}$ and $y=0 \delta(e)$. If $c \delta \alpha(e) \neq B$ or $c \delta(e) \neq B$ put $x=\delta \alpha(e), u=1$ or $y=\delta(e), v=1$ respectively. Then

$$
\chi^{B}(\delta \alpha(e) \delta(e))=u \diamond \chi^{B}(x y) \diamond v \quad \text { and } \quad c(x) \neq B, c(y) \neq B
$$

The elements of $c(x)$ can be listed without repetition as $\overline{1}(x), \overline{1} 1(x), \overline{1} 11(x), \ldots$. Hence

$$
\chi^{B}(x y)=0(x y) \diamond 0(1(x) y) \diamond 0(11(x) y) \diamond \ldots \diamond 1(x y)
$$

is a factorisation of $\chi^{B}(x y)$ by elements of $Y$; there are at most $|c(x)|+2$ factors. Likewise

$$
\begin{gathered}
\chi^{B}\left(\delta^{\prime} \alpha(e) \delta^{\prime}(e)\right)=\widehat{\theta}(u) \diamond \chi^{B}(\theta(x) \theta(y)) \diamond \hat{\theta}(v) \text { and } \\
\chi^{B}(\theta(x) \theta(y))=0(\theta(x) \theta(y)) \diamond 0(1(\theta(x) \theta(y)) \diamond 0(11(\theta(x) \theta(y)) \diamond \ldots \diamond 1(\theta(x) \theta(y)) .
\end{gathered}
$$

By the the induction assumptions and the observations following them, each factor of

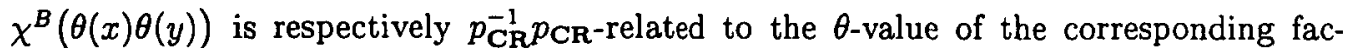
tor of $\chi^{B}(x y)$. So modulo $p_{\mathrm{CR}}^{-1} p_{\mathrm{CR}}$, the factors of $\hat{\theta} \chi^{B}(\delta \alpha(e) \delta(e))$ and $\chi^{B}\left(\delta^{\prime} \alpha(e) \delta^{\prime}(e)\right)$ are identical. Furthermore by induction assumption (ii), any factors of $\widehat{\theta} \chi^{B}(\delta \alpha(e) \delta(e))$ and $\hat{\theta} \chi^{B}(\delta \omega(e))$ are $p_{\mathrm{CR}}^{-1} p_{\mathrm{CR}}$-related if the corresponding factors of $\chi^{B}(\delta \alpha(e) \delta(e))$ and $\chi^{B}(\delta \omega(e))$ are. It follows from Theorem 4.1 that $p_{\mathbf{C R}}\left(\delta^{\prime} \alpha(e) \delta(e)\right)=p_{\mathrm{CR}} \delta^{\prime} \omega(e)$. Also, we saw above that $\varphi=\psi \chi^{B}=\psi \widehat{\theta} \chi^{B}$, so for $z \in \Gamma$ and $c(\delta(z))=B$ we have $\varphi \delta^{\prime}(z)=\psi \chi^{B} \delta(z)_{\theta}=\psi \widehat{\theta} \chi^{B}(\delta(z))=\varphi \delta(z)$.

In the remainder of this paper assume that $\delta$ is as described in Lemma 6.1. We now modify $\Gamma$ and its labellings $\gamma$ and $\delta$ to obtain a graph $\Gamma_{1}$, with labellings $\gamma_{1}$ and $\delta_{1}$ in such a way as to reduce the problem to one in which all vertex labels have content $B$. Let $B=\left\{b_{1}, b_{2}, \ldots, b_{n}\right\}$ and $d=b_{1} b_{2} \ldots b_{n} \in \Omega_{B}^{\kappa} \mathrm{S}$.

Construct $\Gamma_{1}$ from $\Gamma$ as follows. Let

$$
V\left(\Gamma_{1}\right)=V(\Gamma) \cup\{\xi\} \text { and } E\left(\Gamma_{1}\right)=E(\Gamma) \cup\left\{e_{x}: x \in V(\Gamma)\right\}
$$


where $e_{x}$ is a new edge from the new vertex $\xi$ to $x$. Define $\delta_{1}: \Gamma \rightarrow\left(\bar{\Omega}_{B} \mathrm{~S}\right)^{1}$ by

$$
\delta_{1}(x)=\left\{\begin{array}{ll}
d & \text { if } x=\xi \\
d \delta(x) & \text { if } x \in V(\Gamma)
\end{array} \quad \delta_{1}(e)= \begin{cases}\delta(x) & \text { if } e=e_{x} \\
\delta(e) & \text { if } e \in E(\Gamma),\end{cases}\right.
$$

and $\gamma_{1}=\varphi \delta_{1}$. Since $p_{\mathrm{CR}} \delta$ is consistent so is $p_{\mathrm{CR}} \delta_{1}$ by construction, whence $\gamma_{1}$ is a CR-inevitable labelling of $\Gamma_{1}$. The converse is by the following lemma.

Lemma 6.2. Let $\gamma_{1}$ be a CR-inevitable labelling of $\Gamma_{1}$ via $\delta_{1}$ and let $\delta: \Gamma \rightarrow$ $\left(\bar{\Omega}_{B} \mathrm{~S}\right)^{1}$ be given by

$$
\delta(x)=\delta_{1}\left(e_{x}\right) \text { for } x \in V(\Gamma) \text { and } \delta(e)=\delta_{1}(e) \text { for } e \in E(\Gamma) .
$$

If $p_{\mathrm{CR}}(\delta \alpha(e) \cdot \delta(e))$ and $p_{\mathrm{CR}} \delta \omega(e)$ take the same values under the respective functions $c$, 0 and 1 for each $e \in E(\Gamma)$ then $\gamma=\varphi \delta$ is a CR-inevitable labelling of $\Gamma$ via $\delta$.

Proof: This follows from an observation on the completely regular semigroup $\left(\bar{\Omega}_{B} \mathbf{C R}\right)^{1}$. Suppose $u, v \in\left(\bar{\Omega}_{B} \mathbf{C R}\right)^{1}$ are such that $0(u)=0(v), 1(u)=1(v), c(u)=c(v)$ (so $u \mathcal{H} v$ ). Suppose $w \in\left(\bar{\Omega}_{B} \mathbf{C R}\right)^{1}$ and $w u=w v$. We shall prove that this implies $u=v$. If $c(w) \subseteq c(u)$ then $u w u^{\omega} u=u w v^{\omega} v$, and since $u w u^{\omega}=u w v^{\omega}$ and is $\mathcal{H}$-related to $u$ and $v$ then $u=v$. Assume the result whenever $c(w) \subseteq c(d)$ and proceed by induction. If $c(w)=c(d) \notin c(u)$ then there exists $w_{1}, w_{2} \in\left(\bar{\Omega}_{B} \mathbf{C R}\right)^{1}$ such that $w=w_{1} w_{2}$ and $w_{2} u=1(w u)=1(w v)=w_{2} v$; by the assumption then $u=v$.

For each $e \in E(\Gamma)$ we have $p_{\mathbf{C R}}\left(\delta_{1} \alpha(e) \delta_{1}(e)\right)=p_{\mathbf{C R}} \delta_{1} \omega(e)$ so $p_{\mathbf{C R}}(d \delta \alpha(e) \delta(e))=$ $p_{\mathrm{CR}}(d \delta \omega(e))$. Therefore, if $p_{\mathrm{CR}}(\delta \alpha(e) \delta(e))$ and $p_{\mathrm{CR}} \delta \omega(e)$ take the same values under the respective functions $c, 0$ and 1 then by the above $p_{\mathbf{C R}}(\delta \alpha(e) \delta(e))=p_{\mathbf{C R}} \delta \omega(e)$ as required.

We shall construct a graph $\Gamma_{2}$ from $\Gamma_{1}$, labelled by $\left(\bar{\Omega}_{\partial_{B} \bar{\Omega}_{B} S} S d\right)_{\text {Cd }}^{1}$, from which $\chi_{\text {CR,Sd }}^{B}$ can be tested. An observation is needed for this. Suppose $e=(x, y)$ is an edge from $x$ to $y$ in $\Gamma_{1}$. Since $p_{\mathbf{C R}} \delta_{1}$ is consistent then $p_{\mathbf{C R}}\left(\delta_{1}(x) \delta_{1}(e)\right)=p_{\mathbf{C R}} \delta_{1}(y)$. We have $\chi^{B} \delta_{1}(x)=u \diamond 1 \delta_{1}(x)$ for some $u \in\left(\bar{\Omega}_{\partial_{B} \bar{\Omega}_{B} \mathrm{~S}} \mathrm{Sd}\right)_{\mathrm{Cd}}^{1}$ and

$$
\chi^{B}\left(\delta_{1}(x) \delta_{1}(e)\right)= \begin{cases}u \diamond \chi^{B}\left(1 \delta_{1}(x) \cdot 0 \delta_{1}(e)\right) \diamond v & \text { if } c \delta_{1}(e)=B, \text { some } v \in\left(\bar{\Omega}_{\partial_{B} \bar{\Omega}_{B} \mathrm{~S}} \mathrm{Sd}\right)_{\mathrm{Cd}}^{1} \\ u \diamond \chi^{B}\left(1 \delta_{1}(x) \cdot \delta_{1}(e)\right) & \text { if } c \delta_{1}(e) \neq B .\end{cases}
$$

However by Lemma $6.1,1 \delta_{1}(x)$ and $0 \delta_{1}(e)$ or $\delta_{1}(e)$ respectively are in $\Omega_{B}^{\kappa} \mathrm{S}$. So there is a finite $\diamond$-factorisation of $\chi^{B}\left(1 \delta_{1}(x) \cdot 0 \delta_{1}(e)\right)$ or $\chi^{B}\left(1 \delta_{1}(x) \cdot \delta_{1}(e)\right)$ respectively into elements from $\Omega_{B}^{\kappa} \mathrm{S} \cap Y$. Let the factorisation be $u_{1} \diamond u_{2} \diamond \ldots \diamond u_{r}$ for some natural number $r$; call this the $\delta_{1}$-factorisation of $e$ with length $r$.

We can now construct and label $\Gamma_{2}$. Let $\left\{x_{0}: x \in V\left(\Gamma_{1}\right)\right\}$ be a set disjoint from $V\left(\Gamma_{1}\right)$ but of the same size. For each $e=(x, y) \in E\left(\Gamma_{1}\right)$ with $\delta_{1}$-factorisation of length $r$ let graph $\Gamma_{e}$ be as shown. 


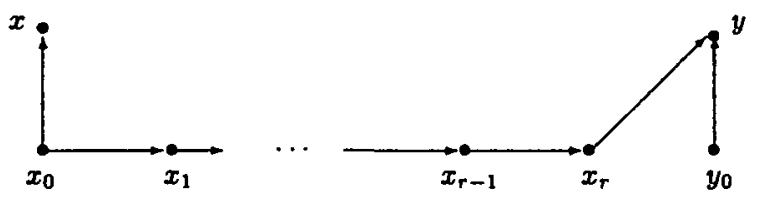

The graph $\Gamma_{2}$ is the union of the graphs $\Gamma_{e}, e \in E\left(\Gamma_{1}\right)$ such that the edges $\left(x_{0}, x\right)$ are identified, for each $x \in V\left(\Gamma_{1}\right)$. Define $\delta_{2}: \Gamma_{2} \rightarrow\left(\bar{\Omega}_{\partial_{B} \bar{\Omega}_{B} \mathrm{~S}} \mathrm{Sd}\right)_{\mathrm{Cd}}^{1}$ as follows. For each $e=(x, y) \in E\left(\Gamma_{1}\right)$, with $\delta_{1}$-factorisation $u_{1} \diamond u_{2} \diamond \ldots \diamond u_{r}$ and $u, v$ defined as above, we label $\Gamma_{e}$ by

$$
\begin{gathered}
\delta_{2}\left(x_{0}\right)=u, \delta_{2}\left(x_{0}, x\right)=1 \delta_{1}(x), \delta_{2}(x)=\chi^{B} \delta_{1}(x)=\delta_{2}\left(x_{0}\right) \diamond \delta_{2}\left(x_{0}, x\right), \\
\delta_{2}\left(x_{i-1}, x_{i}\right)=u_{i}, \delta_{2}\left(x_{i}\right)=\delta_{2}\left(x_{0}\right) \diamond u_{1} \diamond u_{2} \diamond \ldots \diamond u_{i} \text { for } 1 \leqslant i \leqslant r, \\
\delta_{2}\left(x_{r}, y\right)= \begin{cases}v & \text { if } c \delta_{1}(e)=B \\
1 & \text { if } c \delta_{1}(e) \neq B .\end{cases}
\end{gathered}
$$

Label the edge $\left(y_{0}, y\right)$ and its vertices as $\left(x_{0}, x\right)$ and its vertices were labelled, with $y$ replacing $x$. Notice that

$$
\delta_{2}\left(x_{0}\right) \diamond \delta_{2}\left(x_{0}, x_{1}\right) \diamond \ldots \diamond \delta_{2}\left(x_{r-1}, x_{r}\right) \diamond \delta_{2}\left(x_{r}, y\right)=\chi^{B}\left(\delta_{1}(x) \delta_{1}(e)\right)
$$

Since $p_{\mathbf{C R}} \delta_{1}(y)=p_{\mathbf{C R}}\left(\delta_{1}(x) \delta_{1}(e)\right)$ then $\mathbf{G} F \chi_{\mathbf{C R}, \mathbf{S d}}^{B}\left(\delta_{1}(x) \delta_{1}(e)\right)=\chi_{\mathbf{C R}, \mathbf{S d}}^{B} \delta_{1}(y)$ where $\chi_{\mathrm{CR}, \mathrm{Sd}}^{B}=q_{\mathbf{S}, \mathbf{C R}} \chi^{B}$; we have $\chi^{B}\left(\delta_{1}(x) \delta_{1}(e)\right)$ as a o-product of $\delta_{2}$-labels of consecutive edges from $x_{0}$ to $y$ and we have $\chi^{B} \delta_{1}(y)$ as the $\delta_{2}$-label for $y$. Define the labelling $\gamma_{2}: \Gamma_{2} \rightarrow\left(T^{0}, \circ\right)$ by $\gamma_{2}=\psi \delta_{2}$.

Recall that the set $\left(\bar{\Omega}_{\partial_{B} \bar{\Omega}_{B} S} \mathbf{S d}\right)_{C d} \backslash\{0\}$ embeds as a partial subsemigroup in $\bar{\Omega}_{Y} \mathbf{S}$ by the injective partial homomorphism $\mu$ and the canonical homomorphism $\lambda: \bar{\Omega}_{Y} \mathrm{~S} \rightarrow$ $\left(\bar{\Omega}_{\partial_{B} \bar{\Omega}_{B} \mathrm{~S}} \mathrm{Sd}\right)_{\mathrm{Cd}}$ is such that $\lambda \mu$ is the identity map on its domain. As previously noted, the continuous homomorphism $\hat{\theta}$ maps $\left(\bar{\Omega}_{\partial_{B} \bar{\Omega}_{B} \mathrm{~S}} \mathrm{Sd}\right)_{\mathrm{Cd}}$ onto its subsemigroup $\left(\bar{\Omega}_{\theta \partial_{B} \bar{\Omega}_{B} \mathrm{~S}} \mathrm{Sd}\right)_{\mathrm{Cd}}$ and $\left(\bar{\Omega}_{\theta \partial_{B} \bar{\Omega}_{B} \mathbf{S}} \mathbf{S d}\right)_{C d} \backslash\{0\}$ embeds by $\mu$ as a partial subsemigroup in $\bar{\Omega}_{\theta(Y)} \mathbf{S}$. The map $\eta=\mu \widehat{\theta} \delta_{2}$ now relabels $\Gamma_{2}$ by the finitely generated profinite semigroup $\bar{\Omega}_{\theta(Y)} \mathrm{S}$ such that $\lambda \eta=\widehat{\theta} \delta_{2}$.

Let $X=\left\{p_{\mathbf{C R}}^{-1} p_{\mathbf{C R}}(w): w \in \theta(Y)\right\}$ and $\rho: \theta(Y) \rightarrow X$ be given by $\rho(w)=$ $p_{\mathrm{CR}}^{-1} p_{\mathrm{CR}}(w)$. Denote by $\hat{\rho}$ the unique continuous homomorphism that extends $\rho$, as in the diagram.

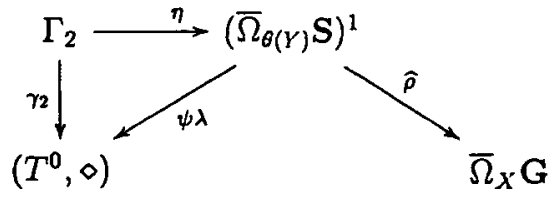

We have seen that $\psi=\psi \widehat{\theta}$. As well, by Lemma 6.1 , it follows that $\mathbf{G} \models$ 
$q_{\mathbf{S}, \mathbf{C R}}^{\mathbf{S d}} \widehat{\theta} \chi^{B}\left(\delta_{1}(x) \delta_{1}(e)\right)=q_{\mathbf{S}, \mathbf{C R}}^{\mathbf{S d}} \widehat{\theta} \chi^{B} \delta_{1}(y)$. Hence by Theorem 4.1 and the construction we get the next Lemma.

LEMMA 6.3. Let $\delta_{1}$ and $\gamma_{1}=\varphi \delta_{1}$ be labellings of $\Gamma_{1}$ by $\bar{\Omega}_{B} \mathrm{~S}$ and $S$ respectively. Let $\eta$ and $\gamma_{2}$ be labellings of $\Gamma_{2}$ by $\bar{\Omega}_{Y} S$ and $\left(T^{0}, \diamond\right)$ respectively as constructed above from $\delta_{1}$ for each $e=(x, y) \in E\left(\Gamma_{1}\right)$. Then $\gamma_{1}$ is CR-inevitable via $\delta_{1}$ if and only if for each $e \in E\left(\Gamma_{1}\right)$ the elements $p_{\mathbf{C R}}\left(\delta_{1} \alpha(e) \cdot \delta_{1}(e)\right)$ and $p_{\mathbf{C R}} \delta_{1} \omega(e)$ take the same values under the respective functions $c, 0$ and 1 , and

$$
\widehat{\rho}\left(\eta\left(x_{0}\right) \eta\left(x_{0}, x_{1}\right) \ldots \eta\left(x_{r-1}, x_{r}\right) \eta\left(x_{r}, y\right)\right)=\widehat{\rho} \eta(y) .
$$

Since $\gamma_{1}$ is a CR-inevitable labelling of $\Gamma_{1}$ then the labelling $\gamma_{2}$ of $\Gamma_{2}$ by $\left(T^{0}, \diamond\right)$ via the labelling $\eta$ of $\Gamma_{2}$ by $\bar{\Omega}_{\theta(Y)} \mathbf{S}$ is $(\mathrm{G}, \hat{\rho})$-inevitable. So we can now apply the strengthened version of Ash's theorem to obtain a labelling $\eta^{\prime}$ of $\Gamma_{2}$ by $\Omega_{\hat{\theta}(Y)}^{\kappa} \mathbf{S}$ via which $\gamma_{2}$ is $(\mathrm{G}, \widehat{\rho})$ inevitable.

However, before we apply the extension of Ash's theorem, we modify the semigroup $\left(T^{0}, \diamond\right)$ so as to ensure that the labelling $\eta^{\prime}$ we obtain is compatible with the labels obtained by Lemma 6.1. We also want to ensure that the labelling $\lambda \eta^{\prime}$ is compatible with $\chi^{B}$; remember that $\lambda \eta=\widehat{\theta} \delta_{2}$ and $\delta_{2}$-labellings are determined from $\chi^{B} \delta_{1}$-labellings. Let $\overline{\overline{1}}(w)$ denote respectively $\overline{1}(w)$ or $\overline{1} 1(w)$ according as $c(w) \neq B$ or $c(w)=B$ and dually define $\overline{\overline{0}}(w)$. Let

$$
R=\left\{(\theta(x), s, \theta(y)) \in \theta(Y) \times T \times \theta(Y):\left\{\begin{array}{l}
\varphi(x)=0(s), \varphi(y)=1(s) \text { if } c(s)=B \\
\varphi(x)=s=\varphi(y) \text { if } c(s) \neq B
\end{array}\right.\right.
$$

and define a binary operation $\square$ on $R \cup\{0\}$ by

$(\theta(x), s, \theta(y)) \square(\theta(u), t, \theta(v))= \begin{cases}(\theta(x), s \diamond t, \theta(v)) & \text { if } \theta(y)=\theta(u), s \diamond t \neq 0 \text { and } \overline{\overline{1}} s \neq \overline{\overline{0}} t \\ 0 & \text { otherwise. }\end{cases}$

Let $\psi^{\prime}:\left(\bar{\Omega}_{\theta \partial_{B} \bar{\Omega}_{B} \mathrm{~S}} \mathrm{Sd}\right)_{\mathrm{Cd}}^{1} \rightarrow\left(R^{0}, \square\right)$ be the unique continuous homomorphism such that for $w \in E\left(\theta \partial_{B} \bar{\Omega}_{B} S\right)$

$$
\psi^{\prime}(w)=(\theta(w), \psi(w), \theta(w))
$$

For $w \in\left(\bar{\Omega}_{\theta \partial_{B} \bar{\Omega}_{B} \mathrm{~S}} \mathrm{Sd}\right)_{\mathrm{Cd}}$ we define $0(w) \in E\left(\theta \partial_{B} \bar{\Omega}_{B} \mathrm{~S}\right)$ to be the initial edge of $w$. Dually define $1(w)$. For $u \in \bar{\Omega}_{Y} \mathrm{~S}$ define $0(u)$ to be the initial term from $Y$ to appear in $u$ and dually define $I(u)$. Notice that since $\delta_{2}$-labellings are determined from $\chi^{B} \delta_{1}$-labellings then $\psi^{\prime} \lambda \eta$-labellings are non-zero

The labelling $\gamma_{2}^{\prime}=\psi^{\prime} \lambda \eta$ of $\Gamma_{2}$ by the finite semigroup $\left(R^{0}, \square\right)$ is $(\mathrm{G}, \widehat{\rho})$-inevitable via the labelling $\eta$ of $\Gamma_{2}$ by the finitely generated $\bar{\Omega}_{\theta(Y)} \mathrm{S}$. Remember that the restriction of $\lambda$ and $\widehat{\theta}$ to $\theta(Y)$ is the identity map on $\theta(Y)$ and that clearly $0 \lambda=0,1 \lambda=1$. So for any labelling $\eta^{\prime}$ of $\Gamma_{2}$ by $\bar{\Omega}_{\theta(Y)} S$ such that $\psi^{\prime} \lambda \eta^{\prime}=\gamma_{2}^{\prime}$, and any $x \in \Gamma_{2}$, if $c \gamma_{2}^{\prime}(x)=B$ 
then $0 \eta^{\prime}(x)=\theta 0 \eta^{\prime}(x)=\theta 0 \eta(x)=0 \eta(x)$ and dually $1 \eta^{\prime}(x)=1 \eta(x)$. If $c \gamma_{2}^{\prime}(x) \neq B$ then $\eta^{\prime}(x) \in \theta(Y)$ so $\eta^{\prime}(x)=\theta \eta^{\prime}(x)=\theta \eta(x)=\eta(x)$.

TheOREM 6.4. If Problem 5.2 has an affirmative answer then $\mathrm{CR}$ is $\kappa$-reducible.

Proof: Assume that $\gamma$ is a CR-inevitable labelling of $\Gamma$ by $S$ that satisfies the conditions of Lemma 6.1. We apply the strengthened version of Ash's theorem to obtain a labelling $\eta^{\prime}$ of $\Gamma_{2}$ by elements of $\Omega_{\theta(Y)}^{\kappa} \mathbf{S}$ for which $\psi^{\prime} \lambda \eta^{\prime}=\gamma_{2}^{\prime}$ is $(\mathbf{G}, \hat{\rho})$-inevitable. We shall assume without loss of generality that $\mu \lambda \eta^{\prime}=\eta^{\prime}$.

Any $w \in \Omega_{\theta(Y)}^{\kappa} S$ has a finite factorisation as a product of terms from $\theta(Y)$. Since $\psi^{\prime} \lambda \eta^{\prime}=\gamma_{2}^{\prime}$ and the range of $\gamma_{2}^{\prime}$ excludes 0 then, by Lemma 3.1 , for any $x \in \Gamma_{2}$ with $c \eta^{\prime}(x)=B$ there exists a unique $u \in \bar{\Omega}_{B}$ S such that $\chi^{B}(u)=\lambda \eta^{\prime}(x)$.

Recall that $\eta=\mu \widehat{\theta} \delta_{2}$ where $\hat{\theta} \delta_{2}=\lambda \eta$ is a labelling of $\Gamma_{2}$ by $\left(\bar{\Omega}_{\theta \theta_{B}} \bar{\Omega}_{B} \mathrm{~S} S \mathrm{Sd}\right)_{\mathrm{Cd}}^{1}$. Consider an edge $e=(x, y) \in E\left(\Gamma_{1}\right)$. The $\delta_{2}$-labels for the associated edges $\left(x_{0}, x\right),\left(y_{0}, y\right)$ and $\left(x_{i-1}, x_{i}\right), 1 \leqslant i \leqslant r$, of $\Gamma_{e}$ are all of content cardinality $|B|-1$. So for each of these edges, $\eta^{\prime}$ and $\eta$ take the same value. As well, since $\hat{\rho} \eta^{\prime}$ is consistent we may assume without loss of generality that $\eta^{\prime}\left(x_{i}\right)=\eta\left(x_{i}\right), 1 \leqslant i \leqslant r$. The remaining elements $z \in \Gamma_{e}$ have content $B$ and so $0 \eta^{\prime}(z)=0 \eta(z)$ and likewise $1 \eta^{\prime}(z)=\eta(z)$.

Let us relabel $\Gamma_{1}$ by $\delta_{1}^{\prime}$, again with $e=(x, y) \in E\left(\Gamma_{1}\right)$. Put

$$
\begin{aligned}
\chi^{B} \delta_{1}^{\prime}(x) & =\lambda \eta^{\prime}(x), \quad \chi^{B} \delta_{1}^{\prime}(y)=\lambda \eta^{\prime}(y) \text { and } \\
\chi^{B} \delta_{1}^{\prime}(e) & =0 \delta_{1}(e) \diamond \lambda \eta^{\prime}\left(x_{r}, y\right) \text { if } c \delta_{1}(e)=B \\
\delta_{1}^{\prime}(e) & =\delta_{1}(e) \text { if } c \delta_{1}(e) \neq B .
\end{aligned}
$$

Since $\psi^{\prime} \lambda \eta^{\prime}=\psi^{\prime} \lambda \eta$, so $\psi \lambda \eta^{\prime}=\psi \lambda \eta$, and since $0 \delta_{1}(e) \diamond \lambda \eta^{\prime}\left(x_{r}, y\right)=\chi^{B} \delta_{1}^{\prime}(e)$ when $c \delta_{1}(e)=$ $B$, we get $\psi \delta_{1}^{\prime}=\gamma_{1}$.

From the above it also follows directly that $0 \delta_{1}^{\prime}(x)=0 \delta_{1}(x), 1 \delta_{1}^{\prime}(x)=1 \delta_{1}(x)$, $0 \delta_{1}^{\prime}(e)=0 \delta_{1}(e)$ and $1 \delta_{1}^{\prime}(e)=1 \delta_{1}(e)$. Therefore to show that $p_{\mathrm{CR}} \delta_{1}^{\prime}$ is consistent it suffices to show that condition 4 of Theorem 4.1 is satisfied. To do this we construct $\delta_{2}^{\prime}$ from $\delta_{1}^{\prime}$ as $\delta_{2}$ was constructed from $\delta_{1}$ and prove that

$$
\widehat{\rho} \mu \widehat{\theta}\left(\delta_{2}^{\prime}\left(x_{0}\right) \diamond \delta_{2}^{\prime}\left(x_{0}, x_{1}\right) \diamond \ldots \diamond \delta_{2}^{\prime}\left(x_{r-1}, x_{r}\right) \diamond \delta_{2}^{\prime}\left(x_{r}, y\right)\right)=\hat{\rho} \mu \hat{\theta} \delta_{2}^{\prime}(y)
$$

Since $1 \delta_{1}^{\prime}(x) \cdot 0 \delta_{1}^{\prime}(e)=1 \delta_{1}(x) \cdot 0 \delta_{1}(e)$ or $1 \delta_{1}^{\prime}(x) \cdot \delta_{1}^{\prime}(e)=1 \delta_{1}(x) \cdot \delta_{1}(e)$ according as $c \delta_{1}(e)=B$ or not, then $\delta_{2}^{\prime}\left(x_{i-1}, x_{i}\right)=\delta_{2}\left(x_{i-1}, x_{i}\right)$ and $\delta_{2}^{\prime}\left(x_{i}\right)=\delta_{2}\left(x_{i}\right), 1 \leqslant i \leqslant r$. So $\delta_{2}^{\prime}\left(x_{i-1}, x_{i}\right)=\lambda \eta^{\prime}\left(x_{i-1}, x_{i}\right)$. We have $\delta_{2}^{\prime}\left(x_{0}\right) \circ 1 \delta_{1}^{\prime}(x)=\delta_{2}^{\prime}(x)=\chi^{B} \delta_{1}^{\prime}(x)=\chi^{B} \lambda \eta^{\prime}(x)=$ $\lambda \eta^{\prime}\left(x_{0}\right) \diamond 1 \delta_{1}^{\prime}(x)$ so we can put $\delta_{2}^{\prime}\left(x_{0}\right)=\lambda \eta^{\prime}\left(x_{0}\right)$. Also $0 \delta_{1}^{\prime}(e) \diamond \delta_{2}^{\prime}\left(x_{r}, y\right)=\delta_{1}^{\prime}(e)=0 \delta_{1}^{\prime}(e) \diamond$ $\lambda \eta^{\prime}\left(x_{r}, y\right)$ so we can put $\delta_{2}^{\prime}\left(x_{r}, y\right)=\lambda \eta^{\prime}\left(x_{r}, y\right)$. Because $\widehat{\theta} \lambda \eta^{\prime}=\lambda \eta^{\prime}$ then

$$
\begin{aligned}
\hat{\rho} \mu \hat{\theta}\left(\delta_{2}^{\prime}\left(x_{0}\right) \diamond \delta_{2}^{\prime}\left(x_{0}, x_{1}\right) \diamond \ldots \diamond \delta_{2}^{\prime}\left(x_{r-1}, x_{r}\right) \diamond \delta_{2}^{\prime}\left(x_{r}, y\right)\right) \\
=\hat{\rho} \mu \lambda\left(\eta^{\prime}\left(x_{0}\right) \cdot \eta^{\prime}\left(x_{0}, x_{1}\right) \cdot \ldots \cdot \eta^{\prime}\left(x_{r-1}, x_{r}\right) \cdot \eta^{\prime}\left(x_{r}, y\right)\right) \\
=\hat{\rho}\left(\eta^{\prime}\left(x_{0}\right) \cdot \eta^{\prime}\left(x_{0}, x_{1}\right) \ldots \eta^{\prime}\left(x_{r-1}, x_{r}\right) \cdot \eta^{\prime}\left(x_{r}, y\right)\right) .
\end{aligned}
$$


But since $\widehat{\rho} \eta^{\prime}$ is consistent, this equals $\widehat{\rho} \eta^{\prime}(y)$. We have $\widehat{\rho} \eta^{\prime}(y)=\widehat{\rho} \mu \lambda \eta^{\prime}(y)=\widehat{\rho} \mu \chi^{B} \delta_{1}^{\prime}(y)=$ $\widehat{\rho} \mu \widehat{\theta} \chi^{B} \delta_{1}^{\prime}(y)=\widehat{\rho} \mu \widehat{\theta} \delta_{2}^{\prime}(y)$.

By the above, we have selected a labelling $\delta_{1}^{\prime}$ of $\Gamma_{1}$ by $\Omega_{B}^{\kappa} \mathrm{S}$ such that $p_{\mathrm{CR}} \delta_{1}^{\prime}$ is consistent and such that for each edge $e \in E\left(\Gamma_{1}\right)$ we have $0 \delta_{1}^{\prime}(e)=0 \delta_{1}(e)$ and $1 \delta_{1}^{\prime}(e)=$ $1 \delta_{1}(e)$, or $\delta_{1}^{\prime}(e)=\delta_{1}(e)$ (according as $c \delta_{1}(e)$ is $B$ or not). The $\delta_{1}$ edge labels of $E\left(\Gamma_{1}\right)$ are the $\delta$ labels for the vertices and edges of $\Gamma$. By Lemma 6.2 we may now select $\delta^{\prime}: \Gamma \rightarrow\left(\Omega_{B}^{\kappa} S\right)^{1}$ from $\delta_{1}^{\prime}$ such that $\varphi \delta^{\prime}=\gamma$ and $\gamma$ is CR-inevitable via $\delta^{\prime}$.

The remainder of the proof is concerned with selecting elements of $\Omega_{B}^{\kappa} S$ to make up a set $P$ that will satisfy the requirements of the induction assumptions when extended to elements of $\Omega_{B}^{\kappa} \mathrm{S}$.

A subset $\left\{d_{1}, \ldots, d_{p}\right\}$ of $S$ is a CR-pointlike subset of $S$ of content $B$ if $c\left(d_{i}\right)=B$ for $1 \leqslant i \leqslant p$ and the labelling $\pi$ of the $p$ vertex chain as shown is CR-inevitable. Here $p \leqslant|S|$.

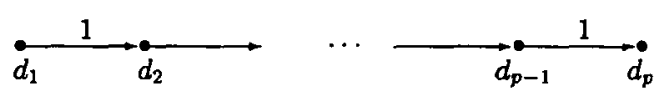

If $\pi$ is CR-inevitable then by the above construction applied to $\pi$ rather than $\gamma$, and by the portion of the proof above, there is a labelling $\varepsilon$ of the chain by $\Omega_{\theta(Y)}^{\kappa} \mathrm{S} \subseteq \Omega_{B}^{\kappa} \mathrm{S}$ such that $\varphi \varepsilon=\pi$ and $p_{\mathbf{C R}} \varepsilon$ is consistent.

Let $\left\{D_{1}, \ldots, D_{q}\right\}$ be the set of all maximal CR-pointlike subsets of $S$ of content $B$, indexed by natural numbers $\leqslant q$. Suppose $D=D_{j}$ for some $j$ and $D=\left\{d_{1}, \ldots, d_{p}\right\}$. So there is a set $R_{D}=\left\{u_{1}, \ldots, u_{p}\right\} \in \Omega_{B}^{\kappa} S$ such that $\varphi\left(u_{i}\right)=d_{i}$ and $p_{\mathbf{C R}}\left(u_{i}\right)=p_{\mathbf{C R}}\left(u_{j}\right)$ for all $i, j, 1 \leqslant i, j \leqslant p$. We shall make an assumption for each $D$ and $u \in R_{D}$. Consider elements $v \in \bar{\Omega}_{B} \mathbf{S}$ such that $\varphi(v)=\varphi(u)$ and recall that $c \varphi=c, 0 \varphi=\varphi 0$ and $1 \varphi=\varphi 1$. If there exists such a $v$ and $v=0(v) v_{1} 1(v)$ for some $v_{1} \in\left(\bar{\Omega}_{B} \mathbf{S}\right)^{1}$ (that is, the 0 and 1 segments of $v$ do not overlap) then we may assume the 0 and 1 segments of $u$ do not overlap. This is because in the last paragraph, if necessary we can modify the labelled graph, and retain CR-inevitability, by adjoining to the vertex labelled $\varphi(v)$ a path labelled as shown

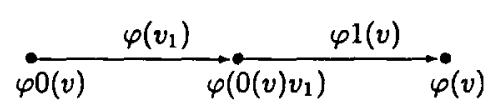

Alternatively if all such $v$ have their 0 and 1 segments overlapping we may assume the 0 and 1 segments of $u$ overlap in a word of minimal content.

As above the function $\chi^{B}$ uniqely provides a finite $\diamond$-factorisation of each $u_{i} \in R_{D}$ into components from $\theta(Y)$. For each $u \in R_{D}$ select $u^{\prime} \in\left(\bar{\Omega}_{\theta \partial_{B}} \bar{\Omega}_{B} \mathrm{~S} S \mathrm{~d}\right)_{C d}^{1}$ such that $\chi^{B} u=0(u) \diamond u^{\prime} \diamond 1(u)$; if $u^{\prime}=1$ then $10(u)=01(u)$.

We now construct $\theta: \bar{\Omega}_{B} \mathrm{~S} \rightarrow P$ to extend $\left.\theta\right|_{U}$ as described in the induction assumption. Suppose $v \in \bar{\Omega}_{B} \mathrm{~S}, c(v)=B$ and let $D_{j}$ be the maximal CR-pointlike subset of $S$ of content $B$ with least index $j$ such that $\varphi p_{\mathrm{CR}}^{-1} p_{\mathrm{CR}}(v) \subseteq D_{j}$. There exists uniquely $u \in R_{D_{\text {, }}}$ 
such that $\varphi(u)=\varphi(v)$. Define $\theta(v)=\theta 0(v) \diamond v^{\prime} \diamond \theta 1(v)$ where $v^{\prime}$ is constructed from $u^{\prime}$ as follows. If $u^{\prime} \neq 1$, let $u^{\prime}$ have $\diamond$-factorisation $u^{\prime}=a_{1} \diamond a_{2} \diamond \ldots \diamond a_{s}$ into $\theta(Y)$-components. For each $i, 1 \leqslant i \leqslant s$, define $x_{i}$ and $y_{i}$ as follows; if $10(u)$ or $01(u)$ or neither is in $\psi^{-1} \psi\left(0\left(a_{i}\right)\right) \cap p_{\mathrm{CR}}^{-1} p_{\mathrm{CR}}\left(0\left(a_{i}\right)\right)$ then respectively let $x_{i}$ be $\theta 10(v)$ or $\theta 01(v)$ or $0\left(a_{i}\right)$, and likewise, if $10(u)$ or $01(u)$ or neither is in $\psi^{-1} \psi\left(1\left(a_{i}\right)\right) \cap p_{\mathbf{C R}}^{-1} p_{\mathbf{C R}}\left(1\left(a_{i}\right)\right)$ then respectively let $y_{i}$ be $\theta 10(v)$ or $\theta 01(v)$ or $1\left(a_{i}\right)$. Then define $b_{i}=a_{i_{x_{i} y_{i}}}$ as determined by induction assumption (iv). Now define $v^{\prime}=b_{1} \diamond b_{2} \diamond \ldots \diamond b_{s}$. Alternatively, if $u^{\prime}=1$ then we may select $v^{\prime}=1$. Without loss of generality we may assume that $\theta(u)=u$ for each $u \in R_{D_{j}}$. Observe that $\varphi\left(a_{i}\right)=\varphi\left(b_{i}\right)$ for each $i$ so $\varphi(v)=\varphi(u)=\varphi\left(\theta(v)\right.$. Also $p_{\mathrm{CR}} a_{i}=p_{\mathrm{CR}} b_{i}$ for each $i$, so modulo $p_{\mathbf{C R}}^{-1} p_{\mathbf{C R}}$, the factorisations of $u$ and $\theta(v)$ are identical except in their first and last terms. If $p_{\mathbf{C R}}(v)=p_{\mathrm{CR}}(w)$ then modulo $p_{\mathbf{C R}}^{-1} p_{\mathbf{C R}}, \theta(v)$ and $\theta(w)$ have identical first and last terms and are constructed from elements of $R_{D_{j}}$ as above, so $p_{\mathbf{C R}} \theta(v)=p_{\mathbf{C R}} \theta(w)$. Define

$$
P=\left\{\theta(v): v \in \bar{\Omega}_{B} \mathbf{S}\right\}
$$

The induction hypothesis (i), (ii) and (iii) can be easily seen to apply for content $B$ terms. With $u \in P$ and $c(u) \in B$, and with $x, y$ as specified in the induction assumption (iv) we can construct $u_{x y}$ by replacing $0(u)$ and $1(u)$ in the $\diamond$-factorisation of $u$ by $x$ and $y$ respectively and by replacing the other factors by compatible factors (as was done above in the construction of $\theta(v))$.

\section{REFERENCES}

[1] J. Almeida, Finite semigroups and universal algebra, Series in Algebra 3, (English translation) (World Scientific, Singapore, 1995).

[2] J. Almeida, 'A syntactical proof of locality of DA', Internat. J. Algebra Comput. 6 (1996), 165-177.

[3] J. Almeida, 'Hyperdecidable pseudovarieties and the calculation of semidirect products', Internat. J. Algebra Comput. 9 (1999), 241-261.

[4] J. Almeida, A. Azevedo, and L. Teixeira, 'On finitely based pseudovarieties of the forms $\mathrm{V} * \mathrm{D}$ and $\mathrm{V} * \mathrm{D}_{n}$ ', J. Pure Appl. Algebra 146 (2000), 1-15.

[5] J. Almeida and M. Delgado, 'Sur certains systèmes d'équations avec contraintes dans un groupe libre', Portugal. Math. 56 (1999), 409-417.

[6] J. Almeida and M. Delgado, 'Sur certains systèmes d'équations avec contraintes dans un groupe libre-addenda', (Technical Report CMUP 1999-22) Univ. Porto, (1999).

[7] J. Almeida and B. Steinberg, 'On the decidability of iterated semidirect products and applications to complexity', Proc. London Math. Soc. 80 (2000), 50-74.

[8] J. Almeida and B. Steinberg, 'Syntactic and global semigroup theory, a synthesis approach', in Algorithmic Problems in Groups and Semigroups, (J. C. Birget, S. W. Margolis, and M. V. Sapir, Editors), Trends Math. (Birkhäuser Boston, Boston, MA, 2000).

[9] J. Almeida and P. G. Trotter, 'Hyperdecidability of pseudovarieties of orthogroups', Glasgow Math. J. (to appear). 
[10] J. Almeida and P. Weil, 'Relatively free profinite monoids: an introduction and examples', in Semigroups, Formal Languages and Groups 466, (J. B. Fountain, Editor) (Kluwer Academic Publ., Dordrecht, 1995), pp. 73-117.

[11] J. Almeida and P. Weil, 'Profinite categories and semidirect products', J. Pure Appl. Algebra 123 (1998), 1-50.

[12] C.J. Ash, 'Inevitable graphs: a proof of the type Il conjecture and some related decision procedures', Internat. J. Algebra Comput. 1 (1991), 127-146.

[13] S. Eilenberg; Automata, Languages and Machines, Vol. $B$ (Academic Press, New York, 1976).

[14] J. Kadourek and L. Polák, 'On the word problem for free completely regular semigroups', Semigroup Forum 34 (1986), 127-138.

[15] F.J. Pastijn and P.G. Trotter, 'Residual finiteness in completely regular semigroup varieties', Semigroup Forum 37 (1988), 127-147.

[16] N.R. Reilly, 'The Rhodes expansion and free objects in varieties of completely regular semigroups', J. Pure Appl. Algebra 69 (1990), 89-109.

[17] J. Rhodes, 'Undecidability, automata and pseudovarieties of finite semigroups', Internat. J. Algebra Comput. 9 (1999), 455-473.

[18] B. Steinberg 'Finite state automata: a geometric approach', Trans. Amer. Math. Soc. (to appear).

[19] P.G. Trotter, 'Free completely regular semigroups', Glasgow Math. J. 25 (1984), 241-254.

Centro de Matemática

Universidade do Porto

P. Gomes Teixeira

4099-002 Porto

Portugal
Department of Mathematics

University of Tasmania

GPO Box 252-37

Hobart, Tas. 7001

Australia 